\title{
The Relation between General Relativity's Metrics and Special Relativity's Gravitational Scalar Generalized Potentials and Case Studies on the Schwarzschild Metric, Teleparallel Gravity, and Newtonian Potential
}

\author{
Spyridon Vossos *(D), Elias Vossos *(D) and Christos G. Massouros * \\ Core Department, Euripus Campus, National and Kapodistrian University of Athens, GR 34400 Euboia, Greece
* Correspondence: svossos@uoa.gr (S.V.); evossos@uoa.gr (E.V.); ChrMas@uoa.gr (C.G.M.)
}

check for updates

Citation: Vossos, S.; Vossos, E.; Massouros, C.G. The Relation between General Relativity's Metrics and Special Relativity's Gravitational Scalar Generalized Potentials and Case Studies on the Schwarzschild Metric, Teleparallel Gravity, and Newtonian Potential. Particles 2021, 4, 536-576. https://doi.org/10.3390/ particles4040039

Academic Editor: Kazuharu Bamba

Received: 29 April 2021

Accepted: 6 August 2021

Published: 8 December 2021

Publisher's Note: MDPI stays neutral with regard to jurisdictional claims in published maps and institutional affiliations.

Copyright: (c) 2021 by the authors. Licensee MDPI, Basel, Switzerland. This article is an open access article distributed under the terms and conditions of the Creative Commons Attribution (CC BY) license (https:/ / creativecommons.org/licenses/by/ $4.0 /)$.

\begin{abstract}
This paper shows that gravitational results of general relativity (GR) can be reached by using special relativity (SR) via a SR Lagrangian that derives from the corresponding GR time dilation and vice versa. It also presents a new SR gravitational central scalar generalized potential $V=V(r, \dot{r}, \dot{\phi})$, where $r$ is the distance from the center of gravity and $\dot{r}, \dot{\phi}$ are the radial and angular velocity, respectively. This is associated with the Schwarzschild GR time dilation from where a SR scalar generalized potential is obtained, which is exactly equivalent to the Schwarzschild metric. Thus, the Precession of Mercury's Perihelion, the Gravitational Deflection of Light, the Shapiro time delay, the Gravitational Red Shift, etc., are explained with the use of SR only. The techniques used in this paper can be applied to any GR spacetime metric, Teleparallel Gravity, etc., in order to obtain the corresponding SR gravitational scalar generalized potential and vice versa. Thus, the case study of Newtonian Gravitational Potential according to SR leads to the corresponding non-Riemannian metric of GR. Finally, it is shown that the mainstream consideration of the Gravitational Red Shift contains two approximations, which are valid in weak gravitational fields only.
\end{abstract}

Keywords: Einstein's Equivalence Principle; gravitational deflection of light; gravitational red shift; kinematics and dynamics of the solar system; linear spacetime transformation; Lorentz metric; precession of Mercury's perihelion; Newtonian gravitational potential; non-Riemannian metric; Schwarzschild metric; Shapiro time delay; Teleparallel Gravity; variable-speed wave

PACS: 02.10.Ud; 03.30.+p; 04.20.-q; 04.80.Cc; 96.12.De; 96.15.De

\section{Introduction}

Throughout this paper, the weak Einstein's Equivalence Principle (EEP) is adopted [1] (p. 245):

$$
m_{G}=m,
$$

where the gravitational mass $\left(m_{\mathrm{G}}\right)$ is equal to the inertial rest mass $(m)$, as it appears in all classical mechanics (Newtonian Physics). Then, the following equality holds, for the gravitational potential energy

$$
U=m_{G} V_{(r)}=m V_{(r)},
$$

where $V_{(r)}$ is the gravitational scalar potential, which depends on the distance $r$ only. In the case of the gravitational scalar generalized potential that is used in this paper, Equation (2) is valid only for the unmoved particles. The following four significant gravitational phenomena are explained below, with both the General Relativity (GR) and the Special Relativity (SR):

(i) Precession of Mercury's Perihelion due to Sun gravity;

(ii) Gravitational Deflection of Light; 
(iii) Shapiro time delay; and

(iv) Gravitational Red Shift.

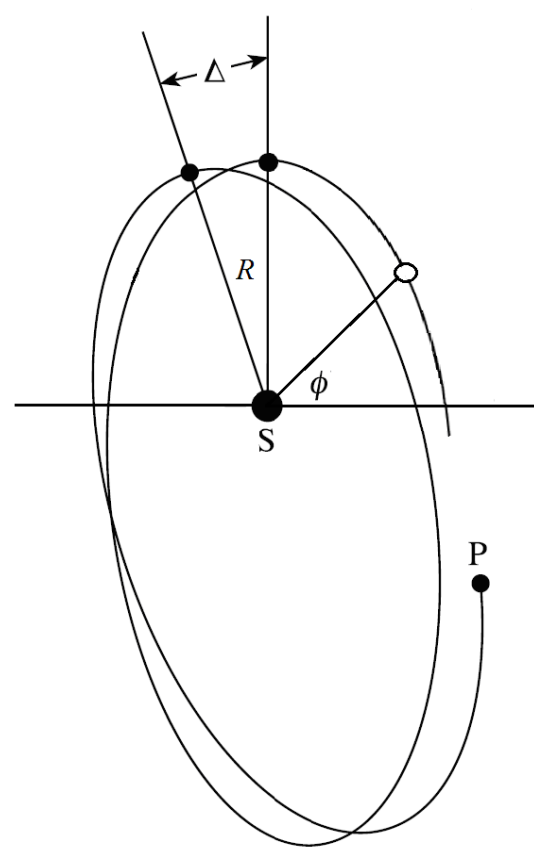

(a)

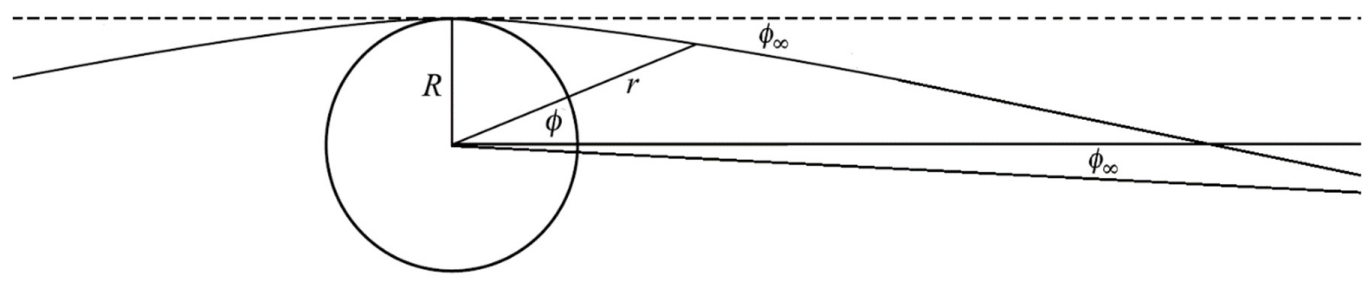

(b)

Figure 1. (a) Precession of the pericenter (perihelion) of a particle/planet due to a spherical mass (the Sun). (b) Gravitational deflection of a ray due to a spherical mass (the Sun). $R$ is the (minimum) distance of the pericenter (perihelion) from the center of gravity, $\Delta$ is the angle that the perihelion precesses per revolution, and $\varphi_{\infty}$ is the half gravitational deflection of a ray.

The Newtonian scalar gravitational potential

$$
V_{N}=-\frac{G M}{r}
$$

combined with the weak Einstein's Equivalence Principle (EEP) (1) and SR provides a method for calculating the precession of Mercury's perihelion due to Sun gravity (Figure 1a), according to which $\Omega=7^{\prime \prime} .16$ per century, [2] (p. 355), [3] (p. 338). This theoretical result though is far from the experimental value: $\Omega_{\exp }=42^{\prime \prime} .9799(9) \mathrm{cy}^{-1}$, which is the contribution of the Sun because of the Schwarzschild gravito-electric effect on the total precession rate of Mercury's perihelion [4] (p. 6), [5] (p. 152), where the Schwarzschild radius

$$
r_{S}=\frac{2 G M}{c^{2}}
$$

is used. 
On the other hand, the gravitational deflection of light (Figure 1b) is an effect that was first predicted by Johann von Soldner in 1801 . He solved the problem by using classical mechanics and the Newtonian gravitational potential (3) [6] (p. 169) in the case of a ray that contains particles (photons) moving with a steady speed $v=c$, which is grazing the Earth (or the Moon, or the Sun). His result for the half deflection $\left(\phi_{\infty}\right)$ was

$$
\tan \phi_{\infty}=\frac{G M}{c \sqrt{c^{2} R^{2}-2 G M R}} \approx \frac{G M}{c^{2} R}=\frac{r_{S}}{2 R^{\prime}}
$$

which gives the magnitude of the total deflection of a ray

$$
\Theta \approx \frac{2 G M}{c \sqrt{c^{2} R^{2}-2 G M R}} \approx \frac{2 G M}{c^{2} R}=\frac{r_{S}}{R^{\prime}}
$$

where $R$ is the minimum distance from the center of gravity. In 1911, Albert Einstein obtained a similar result, before the development of GR. He solved the problem with the use of SR, the EEP, and the Newtonian gravitational potential (3) and he obtained [7] (p. 904):

$$
\Theta=\frac{2 G M}{c^{2} R}=\frac{r_{S}}{R} .
$$

For a ray grazing the Sun, their calculations gave $\Theta=0^{\prime \prime} .84$ and $\Theta=0^{\prime \prime} .83$, respectively. These results are nearly half of the observed value $\Theta_{\exp }=1^{\prime \prime} .75$ [8] (p. 249), which was predicted by the Schwarzschild metric formula [5] (p. 153):

$$
\Theta=\frac{4 G M}{c^{2} R}=\frac{2 r_{S}}{R} .
$$

Generally, potentials that depend on the distance only $\left(V_{\mathrm{SR}}=V_{\mathrm{SR}(r)}\right)$ are inefficient to explain the gravitational deflection of light according to SR and/or classical mechanics, in contrast to the gravitational central scalar generalized potential (160) (see below) according to SR.

The above analysis explains why the gravitational field is usually studied by using Geometric theories of gravitation according to GR [5]. The GR-EEP states that the accelerated motions caused by the gravitational field only (free fall) happen along geodesics of the metric, which corresponds to the particular gravitational field [2] (p. 248).

In this paper, we initially present the Schwarzschild metric and the corresponding Lagrangian, GR time dilation, equations of motion, precession of planets' perihelion, gravitational deflection of light, and the Shapiro time delay. In addition, the corresponding GR generalized potential energy is calculated by reducing the SR kinetic energy from the GR total energy. Thus, it is shown that although the Schwarzschild metric is a static and stationary metric of non-rotating mass, it produces a gravito-magnetic effect because the SR gravitational potential and the SR gravitational relativistic force depend on the velocity of the particle.

The next step is the development of a new method that obtains the GR gravitational results with the use of SR via a SR Lagrangian, which comes from the GR time dilation. Additionally, a new SR gravitational central scalar generalized potential $V=V(r, \dot{r}, \dot{\phi})$ of spherical symmetry is used, where $r$ is the distance from the center of gravity and $\dot{r}, \dot{\phi}$ are the radial and angular velocities, respectively. This method is applied in the case of the Schwarzschild metric of GR and gives the same equations of motion, explaining thus the precession of Mercury's perihelion, gravitational deflection of light, and Shapiro time delay via SR. Moreover, it is shown that the mainstream consideration of gravitational red shift contains two approximations, which are valid for weak gravitational fields only. The alternative SR explanation, which is based on the variable-speed electromagnetic waves around a center of gravity, is presented as well. 
The procedure described in this paper can also be applied to any other GR spacetime metric, such as the Kerr metric or the Kerr-Newman metric, in order to find the corresponding SR gravitational potential.

The above procedure can also be reversed. Thus, if we start from the gravitational central scalar generalized potential (160) (see below) according to SR, then the (Riemannian) Schwarzschild metric is obtained. However, if we start from the Newtonian Gravitational Potential (3) according to SR, then the corresponding metric of GR that is obtained is non-Riemannian (see Section 6). So, the approach described in this paper can also be extended to any theory of gravity (Riemannian and/or non-Riemannian), such as Lorentz Gauge Theory [9-14], Teleparallel Gravity [15-21], etc., with the use of the corresponding time dilation.

In this way, we can work with the gravitational fields in the same manner as in any other type of field (such as the electro-magnetic field) in Minkowski spacetime $\left(\mathrm{M}^{4}\right)$ with the Lorentz metric and we avoid dealing with the motions of particles in the curved spacetime of GR. This is also important for the concept of a unified description of the basic physical interactions, where the biggest barrier is the gravity [9]. This is formulated in the GR curved spacetime, while the other interactions are formulated in $\mathrm{M}^{4}$.

\section{SR: Isometric Linear Transformations in Spacetime Endowed with the Lorentz Metric (Minkowski Spacetime)}

In the case of Relativistic Inertial Observers (RIOs), the representation of the nondegenerate inner product on a holonomic basis $\left[\mathbf{e}_{\mu}\right]=\left[\mathbf{e}_{0}, \mathbf{e}_{1}, \mathbf{e}_{2}, \mathbf{e}_{3}\right]=\left[\mathbf{e}_{c t}, \mathbf{e}_{x}, \mathbf{e}_{y}, \mathbf{e}_{z}\right]$ in $\mathrm{M}^{4}$ is the matrix of the Lorentz Metric

$$
g_{\mathrm{L}}=\operatorname{diag}(-1,1,1,1) .
$$

In addition, SR considers real universal speed

$$
c_{I}=c
$$

and the transformation (Lorentz boost) of a contravariant four-vector is

$$
\mathrm{d} X^{\prime}=\Lambda_{(\beta)} \mathrm{d} X,
$$

where

$$
\Lambda_{(\beta)}=\left[\begin{array}{cc}
\gamma_{(\beta)} & -\gamma_{(\beta)} \beta^{T} \\
-\gamma_{(\beta)} \beta & I_{3}+\frac{\gamma_{(\beta)}-1}{\beta^{T} \beta} \beta \beta^{T}
\end{array}\right] ; \beta^{i}=\frac{\mathrm{d} x^{i}}{\mathrm{~d} x^{0}} ; \beta=\left[\begin{array}{c}
\beta^{1} \\
\beta^{2} \\
\beta^{3}
\end{array}\right]=\left[\begin{array}{c}
\beta_{x} \\
\beta_{y} \\
\beta_{z}
\end{array}\right]
$$

and

$$
\gamma_{(\beta)}=\frac{1}{\sqrt{1-\beta^{T} \beta}}
$$

is the Lorentz $\gamma$-factor (see e.g., [2] p. 24, eq. 1.47). The typical proper Lorentz boost along the $x$-axis (see e.g., [2] p. 21, eq. 1.38) is expressed by the matrix

$$
\Lambda_{(x)(\beta)}=\left[\begin{array}{cccc}
\gamma_{(\beta)} & -\gamma_{(\beta)} \beta & 0 & 0 \\
-\gamma_{(\beta)} \beta & \gamma_{(\beta)} & 0 & 0 \\
0 & 0 & 1 & 0 \\
0 & 0 & 0 & 1
\end{array}\right]
$$


For a Particle $(P)$ with real mass $m$, which is moving with velocity $\vec{v}_{P}=\vec{\beta}_{P} c$ with regard to observer $\mathrm{O}$, the relativistic kinetic energy, the relativistic energy, and the energy of rest mass are, correspondingly:

$$
K=\left(\gamma_{\left(\beta_{P}\right)}-1\right) m c^{2} ; E=\gamma_{\left(\beta_{P}\right)} m c^{2} ; E_{\text {rest }}=m c^{2} .
$$

\section{GR: Metrics with Spherical Symmetry and the Schwarzschild Metric}

\subsection{The Metric of a Static and Centrally Symmetric Gravitational Field}

The single tensor equation $R_{\mu \nu}=0$ comes from Einstein's field equations [8] (pp. 303, 396) applied in vacuum. This produces the metric of a static and centrally symmetric gravitational field

$$
\mathrm{d} S^{2}=-f_{(r)} c^{2} \mathrm{~d} t^{2}+g_{(r)} \mathrm{d} r^{2}+h_{(r)} \mathrm{d} \theta^{2}+h_{(r)} \sin ^{2} \theta \mathrm{d} \phi^{2},
$$

with the following conditions [22] (p. 2):

$$
g_{(r)}=\frac{\mu}{f_{(r)}\left(1-f_{(r)}\right)}\left(\frac{\mathrm{d} f}{\mathrm{~d} r}\right)^{2} ; h_{(r)}=\frac{\mu}{\left(1-f_{(r)}\right)^{2}}
$$

where $\mu$ is an arbitrary constant and $f$ is an arbitrary function of $r$ (not constant). According to Birkhoff's theorem, any metric of spherical symmetry can be reduced to a Schwarzschild metric form, with the use of suitable coordinates [8] (pp. 230-231). It is noted that the coordinates of metric (16) are referred to a Relativistic Inertial Observer with the origin of its frame on the center of gravity O (RIO O). So, they are coordinates of Minkowski spacetime $\left(\mathrm{M}^{4}\right)$.

\subsection{Schwarzschild Metric: Relativistic Potential, Field Strength, and Isotropic Form}

The definition of the original Schwarzschild Relativistic Potential around a center of gravity $\mathrm{O}$ with mass $M$, is

$$
\Phi=\frac{c^{2}}{2} \ln \left(1-\frac{r_{S}}{r}\right)
$$

This is related to the corresponding metric via the formula

$$
\ln f_{(r)}=\frac{2}{c^{2}} \Phi,
$$

which implies

$$
f_{(r)}=1-\frac{r_{S}}{r}
$$

The substitution of the above equation and $\mu=r_{\mathrm{S}}{ }^{2}$ to (17) gives

$$
g_{(r)}=\frac{1}{1-\frac{r_{S}}{r}} ; h_{(r)}=r^{2}
$$

So, we obtain the (Riemannian) original Schwarzschild metric [23]:

$$
\mathrm{d} S^{2}=-\left(1-\frac{r_{S}}{r}\right) c^{2} \mathrm{~d} t^{2}+\frac{1}{1-\frac{r_{S}}{r}} \mathrm{~d} r^{2}+r^{2} \mathrm{~d} \theta^{2}+r^{2} \sin ^{2} \theta \mathrm{d} \phi^{2}
$$


with spatial part

$$
\mathrm{d} l^{2}=\frac{1}{1-\frac{r_{S}}{r}} \mathrm{~d} r^{2}+r^{2} \mathrm{~d} \theta^{2}+r^{2} \sin ^{2} \theta \mathrm{d} \phi^{2} .
$$

In textbooks, the following definition is considered as the radial field strength (e.g., [8] (p. 230)):

$$
\vec{g}=-\vec{\nabla} \Phi=-\frac{\mathrm{d} \Phi}{\mathrm{d} l} \hat{r}=-\frac{\mathrm{d} \Phi}{\mathrm{d} r} \frac{\mathrm{d} r}{\mathrm{~d} l} \hat{r}=-g \hat{r} ; g=\frac{\mathrm{d} \Phi \mathrm{d} r}{\mathrm{~d} r} \frac{\mathrm{d} l}{\mathrm{~d}}
$$

The positive value $(g>0)$ means gravity, while the negative value $(g<0)$ means antigravity. Moreover,

$$
g=\frac{G M}{r^{2}}\left(1-\frac{r_{S}}{r}\right)^{-\frac{1}{2}}>0 .
$$

In the following, it is shown that the field strength on a moving particle is given by a different formula (163), which also contains the velocity of the particle. Formula (25) just gives the field strength of an unmoved particle.

The substitution of

$$
r=\widetilde{r}\left(1+\frac{r_{S}}{4 \widetilde{r}}\right)^{2} \cong \widetilde{r}+\frac{r_{S}}{2} ; \widetilde{r}=\frac{r_{S}}{4}\left(\frac{2 r}{r_{S}}\left(1+\sqrt{1-\frac{r_{S}}{r}}\right)-1\right) \cong r-\frac{r_{S}}{2}
$$

to Schwarzschild metric (22) gives [8] (p. 254):

$$
\mathrm{d} S^{2}=-\left(\frac{1-\frac{r_{S}}{4 \widetilde{r}}}{1+\frac{r_{S}}{4 \widetilde{r}}}\right)^{2} c^{2} \mathrm{~d} t^{2}+\left(1+\frac{r_{S}}{4 \widetilde{r}}\right)^{4}\left(\mathrm{~d} \widetilde{r}^{2}+\widetilde{r}^{2} \mathrm{~d} \theta^{2}+\widetilde{r}^{2} \sin ^{2} \theta \mathrm{d} \phi^{2}\right) .
$$

Besides,

$$
\mathrm{d} \overrightarrow{\widetilde{r}}^{2}=\mathrm{d} \widetilde{r}^{2}+\widetilde{r}^{2} \mathrm{~d} \theta^{2}+\widetilde{r}^{2} \sin ^{2} \theta \mathrm{d} \phi^{2}=\mathrm{d} \widetilde{x}^{2}+\mathrm{d} \widetilde{y}^{2}+\mathrm{d} \widetilde{z}^{2}
$$

where

$$
\widetilde{x}=\widetilde{r} \sin \theta \cos \varphi ; \widetilde{y}=\widetilde{r} \sin \theta \sin \phi ; \widetilde{z}=\widetilde{r} \cos \theta .
$$

So, (27) gives the isotropic form of the Schwarzschild metric [8] (p. 237):

$$
\mathrm{d} S^{2}=-\left(\frac{1-\frac{r_{S}}{4 \widetilde{r}}}{1+\frac{r_{S}}{4 \widetilde{r}}}\right)^{2} c^{2} \mathrm{~d} t^{2}+\left(1+\frac{r_{S}}{4 \widetilde{r}}\right)^{4}\left(\mathrm{~d} \widetilde{x}^{2}+\mathrm{d} \widetilde{y}^{2}+\mathrm{d} \widetilde{z}^{2}\right) .
$$

The approximation in (26) refers to $r \gg r_{S}$. Besides, $r \cong \widetilde{r}$ is a good approximation, because for $r=10 r_{S}$ the relative error is $E_{r}=\frac{\widetilde{r}-r}{r}=-5 \%$, while for $r=100 r_{S}$ it is only $E_{r}=-0.5 \%$.

3.3. Schwarzschild Metric: Lagrangian, Geodesics, Equations of Motion, Equation of Trajectory, Precession of Planets' Perihelion, Deflection of Light, and the Shapiro Time Delay

Consider a particle $P$ with mass $m$, which is moving around the center of gravity $\mathrm{O}$ with mass $M$. The definition of the Lagrangian [8] (p. 205):

$$
L=m \dot{x}^{\mu} g_{\mu v} \dot{x}^{v}
$$


for the orbit on the "plane" $\theta=\pi / 2$, gives the Schwarzschild Lagrangian [8] (p. 238):

$$
L=m\left[-\left(1-\frac{r_{S}}{r}\right) c^{2} \dot{t}^{2}+\frac{1}{1-\frac{r_{S}}{r}} \dot{r}^{2}+r^{2} \dot{\phi}^{2}\right] ; \cdot=\frac{\mathrm{d}}{\mathrm{d} \tau^{\prime}}
$$

where $\tau$ is the proper time, i.e., the time indicated by an arbitrary moving ideal clock. The Euler-Lagrange equations [8] (p. 205):

$$
\frac{\mathrm{d}}{\mathrm{d} \tau}\left(\frac{\partial L}{\partial \dot{x}^{\mu}}\right)-\frac{\partial L}{\partial x^{\mu}}=0 ; \mu=0,1,2
$$

yield the equations of motion:

$$
\begin{aligned}
& E_{G R}=\left(1-\frac{r_{S}}{r}\right) m c^{2} \dot{t} ; \cdot=\frac{\mathrm{d}}{\mathrm{d} \tau} ; \\
& \frac{\mathrm{d}}{\mathrm{d} \tau}\left(\frac{2 \dot{r}}{1-\frac{r_{S}}{r}}\right)-\left[-\frac{r_{S}}{r^{2}} c^{2} \dot{t}^{2}+\frac{\partial}{\partial r}\left(\frac{1}{1-\frac{r_{S}}{r}}\right) \dot{r}^{2}+2 r \dot{\phi}^{2}\right]=0 ; \\
& J_{G R}=m h_{G R}=m r^{2} \dot{\phi} ; \cdot=\frac{\mathrm{d}}{\mathrm{d} \tau},
\end{aligned}
$$

where the integrals of motion are: the GR total energy $\left(E_{\mathrm{GR}}\right)$ and the GR total angular momentum $\left(J_{\mathrm{GR}}\right)$ of the system $\left(h_{\mathrm{GR}}=J_{\mathrm{GR}} / m\right.$ is the GR angular momentum per mass unit), according to RIO O. Lagrangian (32) also satisfies the condition

$$
L=-m c^{2} .
$$

So, the solutions of the above equations of motion can also be used for the practical determination of geodesics [8] (p. 205). Moreover,

$$
h_{G R}=r^{2} \dot{\phi}=r^{2} \frac{\mathrm{d} \phi}{\mathrm{d} \tau} \frac{\mathrm{d} t}{\mathrm{~d} t}=r^{2} \frac{\mathrm{d} \phi}{\mathrm{d} t} \frac{\mathrm{d} t}{\mathrm{~d} \tau}=h_{N} \dot{t} ; h_{N}=r^{2} \frac{\mathrm{d} \phi}{\mathrm{d} t} ;=\frac{\mathrm{d}}{\mathrm{d} \tau^{\prime}}
$$

where $h_{\mathrm{N}}=J_{\mathrm{N}} / m$ is the corresponding Newtonian angular momentum per mass unit. Furthermore, (35) can be written as

$$
2 \ddot{r}\left(1-\frac{r_{S}}{r}\right)-\frac{r_{S}}{r^{2}} \dot{r}^{2}+\left(1-\frac{r_{S}}{r}\right)^{2} \frac{r_{S}}{r^{2}} c^{2} \dot{t}^{2}-2\left(1-\frac{r_{S}}{r}\right)^{2} \dot{\phi}^{2}=0 ; \cdot=\frac{\mathrm{d}}{\mathrm{d} \tau^{\prime}}
$$

or equivalently,

$$
2 \frac{\mathrm{d}^{2} r}{\mathrm{~d} t^{2}}\left(1-\frac{r_{S}}{r}\right)-\frac{r_{S} \dot{r}^{2}}{r^{2}} \frac{\dot{t}^{2}}{t}+\left(1-\frac{r_{S}}{r}\right)^{2} \frac{r_{S}}{r^{2}} c^{2}-2\left(1-\frac{r_{S}}{r}\right)^{2} r \frac{\dot{\phi}^{2}}{\dot{t}^{2}}=0 ;=\frac{\mathrm{d}}{\mathrm{d} \tau} .
$$

Thus, it derives

$$
2 \ddot{r}\left(1-\frac{r_{S}}{r}\right)-\frac{r_{S}}{r^{2}} \dot{r}^{2}+\left(1-\frac{r_{S}}{r}\right)^{2} \frac{r_{S}}{r^{2}} c^{2}-2\left(1-\frac{r_{S}}{r}\right)^{2} r \dot{\phi}^{2}=0 ; \cdot=\frac{\mathrm{d}}{\mathrm{d} t} .
$$


When $\dot{r}=0$, the motion is at the perihelion and/or aphelion or it is a Uniform Circular Motion (UCM) or the particle is momentarily unmoved (for the extra condition $\dot{\phi}=0$ ). So, (41) becomes

$$
2 \ddot{r}+\left(1-\frac{r_{S}}{r}\right) \frac{r_{S}}{r^{2}} c^{2}-2\left(1-\frac{r_{S}}{r}\right) r \dot{\phi}^{2}=0 ; \cdot=\frac{\mathrm{d}}{\mathrm{d} t} .
$$

The UCM (with $r=R=$ const) gives the additional condition $\ddot{r}=0$. Thus, the above equation gives the angular velocity and the centripetal acceleration, respectively:

$$
\omega=\dot{\phi}=\frac{\mathrm{d} \phi}{\mathrm{d} t}=\sqrt{\frac{G M}{R^{3}}} ; a=\frac{v^{2}}{R}=\omega^{2} R=\frac{G M}{R^{2}}=g_{N} .
$$

As is known, the linear relation that connects $\mathrm{N}$ Euler-Lagrange equations yields that a non-constant solution for any (N-1) of them satisfies the Nth as well [8] (p. 213). Since we have already dealt with the case of $r=$ constant, we can now ignore Equation (35) and use Lagrangian (32) combined with condition (37) instead [8] (p. 239). Thus, we have

$$
\dot{r}^{2}=-c^{2}\left(1-\frac{r_{S}}{r}\right)+c^{2}\left(1-\frac{r_{S}}{r}\right)^{2} \dot{t}^{2}-\left(1-\frac{r_{S}}{r}\right) r^{2} \dot{\phi}^{2} ; \cdot=\frac{\mathrm{d}}{\mathrm{d} \tau}
$$

or equivalently,

$$
\left(\frac{\mathrm{d} r}{\mathrm{~d} t}\right)^{2}=-c^{2}\left(1-\frac{r_{S}}{r}\right) \frac{1}{\dot{t}^{2}}+c^{2}\left(1-\frac{r_{S}}{r}\right)^{2}-\left(1-\frac{r_{S}}{r}\right) \frac{r^{2} \dot{\phi}^{2}}{\dot{t}^{2}} ;=\frac{\mathrm{d}}{\mathrm{d} \tau} .
$$

With the use of (34) and (36), the above equations can be written:

$$
\begin{gathered}
\dot{r}^{2}=-c^{2}\left(1-\frac{r_{S}}{r}\right)+\frac{E_{G R^{2}}}{m^{2} c^{2}}-\left(1-\frac{r_{S}}{r}\right) \frac{h_{G R}^{2}}{r^{2}} ;=\frac{\mathrm{d}}{\mathrm{d} \tau^{\prime}} \\
\left(\frac{\mathrm{d} r}{\mathrm{~d} t}\right)^{2}=c^{2}\left(1-\frac{r_{S}}{r}\right)^{2}\left[1-\frac{m^{2} c^{4}}{E_{G R}{ }^{2}}\left(1-\frac{r_{S}}{r}\right)\left(1+\frac{1}{c^{2}} \frac{h_{G R}{ }^{2}}{r^{2}}\right)\right],
\end{gathered}
$$

respectively.

The next step of the study is the determination of the connecting relation of the proper time to the time of RIO O (GR time dilation with respect to RIO O), which is derived below (50). Substituting this into (34), we obtain the final formula of the GR total energy. Finally, the GR generalized potential energy is calculated by reducing the SR relativistic energy from the GR total energy. Thus, we obtain a gravito-electric effect in the case of an unmoved particle, while a moving particle also has a gravito-magnetic effect. So, we conclude that even though the Schwarzschild metric is a static and stationary metric of non-rotating mass, there exists gravito-magnetism because the GR generalized potential energy depends on the velocity of the particle. This is not obvious in the case of GR, because the motion on the curved geodesics is considered as inertial motion. However, Minkowski spacetime endowed with a constant Lorentz metric and null curvature $(K=0)$ makes evident the above consideration as the SR gravitational potential and the SR gravitational force depend on the velocity of the particle as well.

The GR time dilation is obtained from the isometry of spacetime. Thus, (22) is rewritten as

$$
\mathrm{d} S^{2}=-c^{2} \mathrm{~d} \tau^{2}=-\left(1-\frac{r_{S}}{r}\right) c^{2} \mathrm{~d} t^{2}+\frac{1}{1-\frac{r_{S}}{r}} \mathrm{~d} r^{2}+r^{2} \mathrm{~d} \theta^{2}+r^{2} \sin ^{2} \theta \mathrm{d} \phi^{2} ; \theta=\frac{\pi}{2}
$$


or equivalently,

$$
\left(\frac{\mathrm{d} \tau}{\mathrm{d} t}\right)^{2}=1-\frac{r_{S}}{r}-\frac{1}{1-\frac{r_{S}}{r}}\left(\frac{\mathrm{d} r}{\mathrm{~d} t}\right)^{2} \frac{1}{c^{2}}-r^{2}\left(\frac{\mathrm{d} \phi}{\mathrm{d} t}\right)^{2} \frac{1}{c^{2}} ; \theta=\frac{\pi}{2}
$$

This gives the connecting relation of the proper time with the time of RIO O (GR time dilation with respect to RIO O)

$$
\frac{\mathrm{d} t}{\mathrm{~d} \tau}=\left[1-\left(\frac{r_{S}}{r}+\frac{1}{1-\frac{r_{S}}{r}} \frac{\dot{r}^{2}}{c^{2}}+\frac{r^{2} \dot{\phi}^{2}}{c^{2}}\right)\right]^{-\frac{1}{2}} \geq 1 ;=\frac{\mathrm{d}}{\mathrm{d} t}
$$

The corresponding connecting relation of the time of the unmoved observer located at distance $r=r_{0}$ from $O$ (local observer) with the time of RIO O (GR time dilation of the local observer with respect to RIO O) is obtained from the above equation:

$$
\frac{\mathrm{d} t}{\mathrm{~d} t_{\left(r_{0}\right)}}=\left(1-\frac{r_{S}}{r_{0}}\right)^{-\frac{1}{2}} \geq 1
$$

This gives the connecting relation of the proper time to the time of the local observer $t_{\left(r_{0}\right)}$ (GR time dilation with respect to the local observer)

$$
\dot{t}_{\left(r_{0}\right)}=\frac{\mathrm{d} t_{\left(r_{0}\right)}}{\mathrm{d} \tau}=\frac{\mathrm{d} t_{\left(r_{0}\right)}}{\mathrm{d} t} \frac{\mathrm{d} t}{\mathrm{~d} \tau}=\sqrt{1-\frac{r_{S}}{r_{0}}} \dot{t}=\frac{\mathrm{d}}{\mathrm{d} \tau} .
$$

Moreover, the substitution of (50) into (34) gives the final formula of the GR total energy with respect to RIO O

$$
E_{G R}=\frac{1-\frac{r_{S}}{r}}{\sqrt{1-\left(\frac{r_{S}}{r}+\frac{1}{1-\frac{r_{S}}{r}} \bar{c}^{2}+\frac{\dot{r}^{2} \dot{\phi}^{2}}{c^{2}}\right)}} m c^{2} \geq 0 ;=\frac{\mathrm{d}}{\mathrm{d} t} .
$$

Next, the corresponding GR total energy with respect to the unmoved observer located at distance $r=r_{0}$ from $\mathrm{O}$ (the local observer) is obtained as follows:

Schwarzschild Lagrangian (32) is rewritten as

$$
L=m\left[-\left(1-\frac{r_{S}}{r}\right) c^{2}\left(\frac{\mathrm{d} t}{\mathrm{~d} t_{\left(r_{0}\right)}}\right)^{2}\left(\frac{\mathrm{d} t_{\left(r_{0}\right)}}{\mathrm{d} \tau}\right)^{2}+\frac{1}{1-\frac{r_{S}}{r}} \dot{r}^{2}+r^{2} \dot{\phi}^{2}\right] ; \cdot=\frac{\mathrm{d}}{\mathrm{d} \tau^{\prime}}
$$

where coordinate time is the local time $t_{\left(r_{0}\right)}$. The application of the corresponding EulerLagrange equation (33) for $\mu=0$ yields

$$
E_{G R\left(r_{0}\right)}=\left(1-\frac{r_{S}}{r}\right) m c^{2}\left(\frac{\mathrm{d} t}{\mathrm{~d} t_{\left(r_{0}\right)}}\right)^{2} \frac{\mathrm{d} t_{\left(r_{0}\right)}}{\mathrm{d} \tau}
$$


which, with the use of (51) and (52), is transformed to

$$
E_{G R\left(r_{0}\right)}=\frac{1-\frac{r_{S}}{r}}{1-\frac{r_{S}}{r_{0}}} m c^{2} \sqrt{1-\frac{r_{S}}{r_{0}}} \dot{i} ; \cdot=\frac{\mathrm{d}}{\mathrm{d} \tau} .
$$

This combined with (34) gives

$$
E_{G R\left(r_{0}\right)}=\left(1-\frac{r_{S}}{r_{0}}\right)^{-\frac{1}{2}} E_{G R},
$$

which relates the GR total energy with respect to the local observer to the corresponding one of the RIO O.

Now, considering zero kinetic energy $(K=0)$, when the particle $P$ is static $\left(\beta_{\mathrm{p}}=0\right)$, $E_{G R\left(\beta_{P}=0\right)}=E_{\text {rest }}+U_{(r)}$, where $U_{(r)}$ is the potential energy of the unmoved particle. Substituting (15iii) and (51) into the above equation, we have

$$
U_{(r)}=\left(\sqrt{1-\frac{r_{S}}{r}}-1\right) m c^{2} \leq 0 .
$$

This, combined with (2), gives the Schwarzschild Potential of the unmoved particle

$$
V_{(r)}=\left(\sqrt{1-\frac{r_{S}}{r}}-1\right) c^{2} \leq 0,
$$

with respect to RIO O. The above is the central potential with field strength

$$
\vec{g}_{(r)}=-\frac{d V}{d r} \hat{r}=-\frac{G M}{r^{2}}\left(1-\frac{r_{s}}{r}\right)^{-\frac{1}{2}} \hat{r},
$$

which is the same as (25). Additionally, the mechanic energy $E_{\mathrm{m}}=E_{\mathrm{GR}}-E_{\mathrm{rest}}=K+U_{\mathrm{g}}$ with respect to RIO O is calculated:

$$
E_{m}=\left(\frac{1-\frac{r_{S}}{r}}{\sqrt{1-\left(\frac{r_{S}}{r}+\frac{1}{1-\frac{r_{S}}{r} c^{2}}+\frac{r^{2} \dot{\phi}^{2}}{c^{2}}\right)}}-1\right) m c^{2} ; \cdot=\frac{\mathrm{d}}{\mathrm{d} t} .
$$

Moreover, the GR generalized potential energy is defined as

$$
U_{g}=E_{\mathrm{GR}}-E_{\text {rest }}-K=E_{\mathrm{GR}}-E .
$$

The consideration of the SR relativistic energy (15ii) gives

$$
U_{g}=\left(\left(1-\frac{r_{S}}{r}\right)\left[1-\left(\frac{r_{S}}{r}+\frac{1}{1-\frac{r_{S}}{r}} \bar{c}^{2}+\frac{r^{2} \dot{\phi}^{2}}{c^{2}}\right)\right]^{-\frac{1}{2}}-\gamma_{\left(\beta_{P}\right)}\right) m c^{2}
$$


with respect to RIO O. We also observe that if $\beta_{P} \rightarrow 0$, the above equation coincides with (58).

Finally, the substitution of (50) into (38i) gives

$$
h_{G R}=h_{N} \frac{\mathrm{d} t}{\mathrm{~d} \tau}=h_{N}\left[1-\left(\frac{r_{S}}{r}+\frac{1}{1-\frac{r_{S}}{r}} \frac{\dot{r}^{2}}{c^{2}}+\frac{r^{2} \dot{\phi}^{2}}{c^{2}}\right)\right]^{-\frac{1}{2}} \geq h_{N} ; h_{N}=r^{2} \frac{\mathrm{d} \phi}{\mathrm{d} t} ; \cdot=\frac{\mathrm{d}}{\mathrm{d} t} .
$$

In the case of a particle or planet at the perihelion and/or aphelion, or in UCM (where $r=R ; \dot{r}=0)$, the above equation becomes

$$
h_{G R}=h_{N}\left[1-\left(\frac{r_{S}}{R}+\frac{R^{2} \dot{\phi}_{(R)}^{2}}{c^{2}}\right)\right]^{-\frac{1}{2}} \geq h_{N} ; h_{N}=r^{2} \frac{d \phi}{\mathrm{d} t} ; \cdot=\frac{\mathrm{d}}{\mathrm{d} t}
$$

More specifically, for a particle or planet in UCM, we obtain

$$
h_{G R}=h_{N}\left[1-\left(\frac{r_{S}}{R}+\frac{G M}{c^{2} R}\right)\right]^{-\frac{1}{2}}=h_{N}\left[1-\frac{3 r_{S}}{2 R}\right]^{-\frac{1}{2}} \geq h_{N} ; h_{N}=r^{2} \frac{\mathrm{d} \phi}{\mathrm{d} t} ; \cdot=\frac{\mathrm{d}}{\mathrm{d} t^{\prime}}
$$

where (43i) has been applied in the process.

In the case of a photon $(m=0)$, the velocity at an infinite distance from the center of gravity $\mathrm{O}$ is $c_{p}=c_{l}=c$. Additionally, according to (36), the total angular momentum of the system $J_{G R}=m r^{2} \dot{\phi}=m h_{G R}$ is generally finite and nonzero (except for radial motion). So,

$$
h_{G R}=+\infty,
$$

and (63) gives

$$
1-\left(\frac{r_{S}}{r}+\frac{1}{1-\frac{r_{S}}{r}} \frac{\dot{r}^{2}}{c^{2}}+\frac{r^{2} \dot{\phi}^{2}}{c^{2}}\right)=0 ; \cdot=\frac{\mathrm{d}}{\mathrm{d} t} .
$$

This can also be obtained from the energy formula (53), when considering $E \neq 0$. We observe that the above equation relates the radial to the angular velocity of a photon located at distance $r$. Furthermore, the speed of a photon $\left(c_{p}\right)$ is given by the formula

$$
c_{P}^{2}=\dot{r}^{2}+r^{2} \dot{\phi}^{2}
$$

Thus, (67) becomes

$$
1-\left(\frac{r_{S}}{r}+\frac{1}{1-\frac{r_{S}}{r} c^{2}}+\frac{\dot{r}^{2}}{c^{2}}\right)=0
$$

or equivalently,

$$
1-\left(\frac{r_{S}}{r}+\frac{\frac{r_{S}}{r}}{1-\frac{r_{S}}{r}} \frac{\dot{r}^{2}}{c^{2}}+\frac{c_{P}^{2}}{c^{2}}\right)=0
$$


In the case of a photon in radial motion, it is $c_{p}=\dot{r}$ and (69) gives

$$
c_{p}= \pm\left(1-\frac{r_{S}}{r}\right) c ; \gamma_{\left(\beta_{p}\right)}=\frac{1}{\sqrt{\frac{r_{S}}{r}\left(2-\frac{r_{S}}{r}\right)}}
$$

where the \pm sign signifies whether the photon moves away from $\mathrm{O}$ or approaches it. From (71), it derives that when $r=r_{S}$, i.e., on the horizon the speed of light is zero $\left(c_{p}=0\right)$, while as the distance from $\mathrm{O}$ becomes extremely large, $\left|c_{p}\right| \rightarrow c$ and the Lorentz $\gamma$-factor approaches infinity. In the case of motion at the perihelion/aphelion or UCM, where $r=R$, $\dot{r}=0$, the photon speed is denoted by $c_{R}$ and (70) becomes

$$
1-\left(\frac{r_{S}}{R}+\frac{c_{R}^{2}}{c^{2}}\right)=0,
$$

or equivalently,

$$
c_{R}=c \sqrt{1-\frac{r_{S}}{R}}
$$

The comparison of formulas (71i) and (73) yields that at the same distance from $\mathrm{O}$, the speed of a photon in radial motion is smaller than the corresponding speed at the perihelion/aphelion or UCM. Moreover, the combination of (65) and (66) gives the corresponding radius of $\mathrm{UCM}$ :

$$
R=\frac{3}{2} r_{S}
$$

The above result can also be extracted by substituting (43i) into Schwarzschild metric (22) [8] (p. 239). Furthermore, the substitution of (74) into (73) gives

$$
c_{R}=c \sqrt{1-\frac{2}{3}}=\frac{1}{\sqrt{3}} c .
$$

The orbit of motion [8] (pp. 241-245) derives from the exact differential equation of orbit

$$
\frac{\mathrm{d}^{2} u}{\mathrm{~d} \phi^{2}}+u=\frac{G M}{h_{G R}{ }^{2}}+3 \frac{G M}{c^{2}} u^{2} ; u=\frac{1}{r} ; h_{G R}=r^{2} \dot{\phi} ;=\frac{\mathrm{d}}{\mathrm{d} \tau}
$$

This presents a similarity to the orbit of a conic section with differential equation

$$
\frac{\mathrm{d}^{2} u}{\mathrm{~d} \phi^{2}}+u=\frac{1}{R(1+e)}=\frac{1}{a\left|1-e^{2}\right|}=\frac{G M}{h_{G R}^{2}},
$$

whose solution

$$
u=\frac{1}{r}=\frac{1+e \sin \phi}{R(1+e)}=\frac{1+e \sin \phi}{a\left|1-e^{2}\right|}=\frac{G M}{h_{G R^{2}}}(1+e \sin \phi),
$$

where $R$ is the (minimum) distance of the pericenter (perihelion) from the center of gravity $\mathrm{O}, e$ is the eccentricity, $\alpha$ is the semimajor axis of the conic section, and the angle $\phi$ is measured from the axis that passes through the center of gravity and is perpendicular to the radius of the perihelion $R$ as is shown in Figure 1. It is noted that

$$
R=a|1-e| ; \frac{h_{G R}^{2}}{G M}=R(1+e)=a\left|1-e^{2}\right| .
$$


When the velocities are small with respect to $c\left(u \ll c\right.$ or $\left.r \gg r_{S}\right)$, we can substitute the solution of the simplified differential equation (77) into the last term of the exact differential equation of motion (76i). This yields the approximate differential equation of motion (which only approximates the UCM):

$$
\frac{\mathrm{d}^{2} u}{\mathrm{~d} \phi^{2}}+u=\frac{G M}{h_{G R}{ }^{2}}+3 \frac{G^{3} M^{3}}{c^{2} h_{G R}{ }^{4}}(1+e \sin \phi)^{2} ; u=\frac{1}{r^{\prime}} ; h_{G R}=r^{2} \dot{\phi} ; \cdot=\frac{\mathrm{d}}{\mathrm{d} \tau^{\prime}}
$$

with exact solution

$$
\begin{aligned}
u & =\frac{G M}{h_{G R^{2}}}\left(1+e \sin \phi+3 \frac{G^{2} M^{2}}{c^{2} h_{G R^{2}}} e\left(\frac{\pi}{2}-\phi\right) \cos \phi\right) ; h_{G R}=r^{2} \dot{\phi} ;=\frac{\mathrm{d}}{\mathrm{d} t^{\prime}} \\
\frac{G M}{h_{G R}{ }^{2}} & =\frac{1}{R(1+e)}=\frac{1}{a\left|1-e^{2}\right|} .
\end{aligned}
$$

The approximate solution of (79) is obtained if we rewrite (80i) as

$$
u=\frac{G M}{h_{G R}{ }^{2}}\left[1+e\left(\sin \phi+3 \frac{G^{2} M^{2}}{c^{2} h_{G R}^{2}}\left(\frac{\pi}{2}-\phi\right) \cos \phi\right)\right]
$$

and use the identity

$$
\sin (\phi+d)=\sin \phi \cos d+\cos \phi \sin d .
$$

These are associated by using

$$
\begin{gathered}
d=\frac{3 G^{2} M^{2}}{c^{2} h_{G R}{ }^{2}}\left(\frac{\pi}{2}-\phi\right)=\frac{3 G M}{c^{2} R(1+e)}\left(\frac{\pi}{2}-\phi\right)=\frac{3 G M}{c^{2} a\left|1-e^{2}\right|}\left(\frac{\pi}{2}-\phi\right) \ll 1 ; \\
\cos d \approx 1 ; \sin d \approx d .
\end{gathered}
$$

Thus, we have

$$
\begin{aligned}
u & =\frac{G M}{h_{G R}{ }^{2}}\left[1+e \sin \left(\phi+\frac{3 G^{2} M^{2}}{c^{2} h_{G R^{2}}}\left(\frac{\pi}{2}-\phi\right)\right)\right]= \\
& =\frac{G M}{h_{G R}{ }^{2}}\left[1+e \sin \left(\phi\left(1-3 \frac{G^{2} M^{2}}{c^{2} h_{G R^{2}}}\right)+\frac{3 \pi G^{2} M^{2}}{2 c^{2} h_{G R}{ }^{2}}\right)\right],
\end{aligned}
$$

which can be written as

$$
\begin{aligned}
u & \approx \frac{G M}{h_{G R}^{2}}\left[1+e \sin \left(\lambda_{G R} \phi+\left(1-\lambda_{G R}\right) \frac{\pi}{2}\right)\right] \\
\lambda_{G R} & =1-\frac{3 G^{2} M^{2}}{c^{2} h_{G R}^{2}}=1-\frac{3 G M}{c^{2} R(1+e)}=1-\frac{3 G M}{c^{2} a\left(1-e^{2}\right)^{\prime}}
\end{aligned}
$$

or equivalently,

$$
u=\frac{1}{r} \approx \frac{G M}{h_{G R}^{2}}\left[1+e \sin \left(\lambda_{G R}\left(\phi-\frac{\pi}{2}\right)+\frac{\pi}{2}\right)\right] .
$$


This gives

$$
\begin{aligned}
u & =\frac{1}{r} \approx \frac{G M}{h_{G R}{ }^{2}}\left[1+e \cos \left(\lambda_{G R}\left(\phi-\frac{\pi}{2}\right)\right)\right]= \\
& =\frac{1}{R(1+e)}\left[1+e \cos \left(\lambda_{G R}\left(\phi-\frac{\pi}{2}\right)\right)\right]= \\
& =\frac{1}{a\left|1-e^{2}\right|}\left[1+e \cos \left(\lambda_{G R}\left(\phi-\frac{\pi}{2}\right)\right)\right]
\end{aligned}
$$

where

$$
\lambda_{G R}=1-\frac{3 G^{2} M^{2}}{c^{2} h_{G R}{ }^{2}}=1-\frac{3 G M}{c^{2} R(1+e)}=1-\frac{3 G M}{c^{2} a\left(1-e^{2}\right)}
$$

under the restriction

$$
0<\frac{6 \pi G^{2} M^{2}}{c^{2} h_{G R}{ }^{2}}=\frac{6 \pi G M}{c^{2} R(1+e)}=\frac{6 \pi G M}{c^{2} a\left|1-e^{2}\right|} \ll 1 .
$$

In the case of ellipse $(0<e<1)$, every perihelion has

$$
\cos \left(\lambda_{G R}\left(\phi-\frac{\pi}{2}\right)\right)=1
$$

The first, the second, and the $n$-th perihelion correspond to $\phi=\frac{\pi}{2}, \phi=\frac{2 \pi}{\lambda_{G R}}+\frac{\pi}{2}$, and $\phi=\frac{2 n \pi}{\lambda_{G R}}+\frac{\pi}{2}$, respectively (Figure 1a). Thus, the orbit can be regarded as a conic section that rotates ("precesses") about focus $\mathrm{O}$ :

$$
\begin{aligned}
\Delta & =\frac{2 \pi}{\lambda_{G R}}-2 \pi=\left(\frac{1}{\lambda_{G R}}-1\right) 2 \pi \approx 2 \pi\left(1-\lambda_{G R}\right)= \\
& =\frac{6 \pi G^{2} M^{2}}{c^{2} h_{\mathrm{GR}}^{2}}=\frac{6 \pi G M}{R(1+e) c^{2}}=\frac{6 \pi G M}{a\left(1-e^{2}\right) c^{2}}
\end{aligned}
$$

rad per revolution. Observe that the above equation also predicts the precession of cycle $(e=0)$, because it derives from the approximate solution (87) of the approximate differential equation of motion (79). Moreover, the angular velocity of rotation of pericenter (perihelion) is given by the formula

$$
\Omega\left(\frac{\prime \prime}{\mathrm{cy}}\right)=\Delta\left(\frac{\mathrm{rad}}{\mathrm{rev}}\right)\left(\frac{360^{\circ}}{2 \pi \mathrm{rad}}\right)\left(\frac{3600^{\prime \prime}}{1^{\circ}}\right) \frac{1}{T}\left(\frac{\mathrm{rev}}{\mathrm{day}}\right)\left(\frac{365.242 \text { day }}{\text { year }}\right)\left(\frac{100 \text { year }}{\mathrm{cy}}\right),
$$

or equivalently,

$$
\Omega\left(\frac{\prime \prime}{\mathrm{cy}}\right)=\Delta\left(\frac{\mathrm{rad}}{\mathrm{rev}}\right)\left(\frac{7533657 \times 10^{3^{\prime \prime}} \cdot \mathrm{day}}{\mathrm{rad} \cdot \mathrm{cy}}\right) \frac{1}{T}\left(\frac{\mathrm{rev}}{\mathrm{day}}\right) .
$$

The corresponding angular and radial velocities of a particle (planet) are obtained via the following process. We initially calculate $E_{\mathrm{GR}}=E_{\mathrm{GR}}(R, e)$ and $h_{\mathrm{GR}}=h_{\mathrm{GR}}(R, e)$ by working 
on the first pericenter (perihelion) of the ellipse (there exists only one in a parabola and hyperbola), where $\phi=\frac{\pi}{2} ; R=\alpha|1-e| ; \dot{r}=0 ; \ddot{\phi}=0$. Thus, (53) and (64) become

$$
\begin{aligned}
& E_{G R}=\frac{1-\frac{r_{S}}{R}}{\sqrt{1-\left(\frac{r_{S}}{R}+\frac{R^{2} \dot{\phi}_{(R)}^{2}}{c^{2}}\right)} m c^{2} \geq 0} \\
& h_{G R}=R^{2} \dot{\phi}_{(R)}\left[1-\left(\frac{r_{S}}{R}+\frac{R^{2} \dot{\phi}_{(R)}^{2}}{c^{2}}\right)\right]^{-\frac{1}{2}} ;=\frac{\mathrm{d}}{\mathrm{d} t} .
\end{aligned}
$$

The null radial velocity at the perihelion transforms (47) to

$$
1-\frac{m^{2} c^{4}}{E_{G R}{ }^{2}}\left(1-\frac{r_{S}}{R}\right)\left(1+\frac{1}{c^{2}} \frac{h_{G R}^{2}}{R^{2}}\right)=0 .
$$

Moreover, (95), with the use of (94) becomes

$$
1-\frac{1-\left(\frac{r_{S}}{R}+\frac{R^{2} \dot{\phi}_{(R)}^{2}}{c^{2}}\right)}{\left(1-\frac{r_{S}}{R}\right)}\left(1+\frac{1}{c^{2}} \frac{R^{2} \dot{\phi}_{(R)}^{2}}{1-\left(\frac{r_{S}}{R}+\frac{R^{2} \dot{\phi}_{(R)}{ }^{2}}{c^{2}}\right)}\right)=0,
$$

or equivalently,

$$
1-\frac{1}{\left(1-\frac{r_{S}}{R}\right)}\left(1-\left(\frac{r_{S}}{R}+\frac{R^{2} \dot{\phi}_{(R)}^{2}}{c^{2}}\right)+\frac{1}{c^{2}} R^{2} \dot{\phi}_{(R)}^{2}\right)=0,
$$

which is identity for any value of $\dot{\phi}_{(R)}$ and $R$. So, a different approach with differentiation of (87) with respect to time will be followed:

$$
\begin{aligned}
\frac{\dot{r}}{r^{2}}= & \frac{G M e}{h_{G R}{ }^{2}} \lambda_{G R} \dot{\phi} \sin \left(\lambda_{G R}\left(\phi-\frac{\pi}{2}\right)\right)= \\
= & \frac{e}{R(1+e)} \lambda_{G R} \dot{\phi} \sin \left(\lambda_{G R}\left(\phi-\frac{\pi}{2}\right)\right)= \\
= & \frac{e}{a\left(1-e^{2}\right)} \lambda_{G R} \dot{\phi} \sin \left(\lambda_{G R}\left(\phi-\frac{\pi}{2}\right)\right) \\
\frac{\ddot{r} r^{2}-2 r \dot{r}^{2}}{r^{4}}= & \frac{G M e}{h_{\mathrm{GR}}^{2}} \lambda_{G R}\left[\lambda_{G R} \dot{\phi}^{2} \cos \left(\lambda_{G R}\left(\phi-\frac{\pi}{2}\right)\right)+\right. \\
& \left.+\ddot{\phi} \sin \left(\lambda_{G R}\left(\phi-\frac{\pi}{2}\right)\right)\right] ;=\frac{\mathrm{d}}{\mathrm{d} t} .
\end{aligned}
$$


At the perihelion $(\dot{r}=0, \ddot{\phi}=0)$, the above equation becomes

$$
\frac{\ddot{r}_{(R)}}{R^{2}}=\frac{G M e}{h_{G R}{ }^{2}} \lambda_{G R}^{2} \dot{\phi}_{(R)}^{2} .
$$

Moreover, the combination of (78ii) with (94ii) gives

$$
h_{G R}{ }^{2}=G M R(1+e)=G M a\left|1-e^{2}\right|=\frac{R^{4} \dot{\phi}_{(R)}{ }^{2}}{1-\left(\frac{r_{S}}{R}+\frac{R^{2} \dot{\phi}_{(R)}{ }^{2}}{c^{2}}\right)} ; \cdot=\frac{\mathrm{d}}{\mathrm{d} t^{\prime}}
$$

which yields

$$
\begin{aligned}
& {\left[1-\left(\frac{r_{S}}{R}+\frac{R^{2} \dot{\phi}_{(R)}^{2}}{c^{2}}\right)\right] G M R(1+e)=} \\
& =\left[1-\left(\frac{r_{S}}{R}+\frac{R^{2} \dot{\phi}_{(R)}^{2}}{c^{2}}\right)\right] G M a\left|1-e^{2}\right|=R^{4} \dot{\phi}_{(R)}{ }^{2},
\end{aligned}
$$

or equivalently,

$$
\dot{\phi}_{(R)}=\sqrt{\frac{\left(1-\frac{r_{S}}{R}\right) G M R(1+e)}{R^{4}+\frac{r_{S} R^{3}}{2}(1+e)}} ; R=a|1-e| \gg r_{S} .
$$

Furthermore, the GR total energy can be calculated by substituting (103) into (94i):

$$
E_{G R}=\frac{1-\frac{r_{S}}{R}}{\sqrt{1-\left(\frac{r_{S}}{R}+\frac{r_{S}}{2 R} \frac{\left(1-\frac{r_{S}}{R}\right)(1+e)}{1+\frac{r_{S}}{2 R}(1+e)}\right)}} m c^{2} \geq 0 ; R=a|1-e| \gg r_{S},
$$

or equivalently,

$$
E_{G R}=\frac{1-\frac{r_{S}}{R}}{\sqrt{1-\frac{r_{S}}{2 R}\left(\frac{2+\frac{r_{S}}{R}(1+e)+\left(1-\frac{r_{S}}{R}\right)(1+e)}{1+\frac{r_{S}}{2 R}(1+e)}\right)}} m c^{2} \geq 0 ; R=a|1-e| \gg r_{S} .
$$

Thus, we obtain

$$
E_{G R}=\frac{1-\frac{r_{S}}{R}}{\sqrt{1-\frac{r_{S}}{2 R}\left(\frac{3+e}{1+\frac{r_{S}}{2 R}(1+e)}\right)}} m c^{2} \geq 0 ; R=a|1-e| \gg r_{S}
$$


and the mechanical energy (61) becomes

$$
E_{m}=\left[\left(1-\frac{r_{S}}{R}\right)\left(1-\frac{r_{S}}{2 R} \cdot \frac{3+e}{1+\frac{r_{S}}{2 R}(1+e)}\right)^{-\frac{1}{2}}-1\right] m c^{2} ; R=a|1-e| \gg r_{S} .
$$

In the case of the $\operatorname{UCM}(e \rightarrow 0, a \rightarrow R),(103)$ gives the angular velocity

$$
\dot{\phi}_{(R)}=\sqrt{\frac{\left(1-\frac{r_{S}}{R}\right) G M R}{R^{4}+\frac{r_{S} R^{3}}{2}}},
$$

which is slightly smaller than the valid one given by (43i), as it derives from the approximate solution (87) of the approximate differential equation of motion (79).

Moreover, the gravitational deflection of light can be obtained as follows [8] (pp. 24849). Initially, (66) turns (76) to

$$
\frac{\mathrm{d}^{2} u}{\mathrm{~d} \phi^{2}}+u=3 \frac{G M}{c^{2}} u^{2} ; u=\frac{1}{r} .
$$

In the case of large distances from the center of gravity with respect to $r_{S}\left(r \gg r_{S} ; u \ll 1 / r_{S}\right)$, we can substitute the solution (straight line) of the simplified equation of orbit:

$$
\frac{\mathrm{d}^{2} u}{\mathrm{~d} \phi^{2}}+u=0 ; u=\frac{\sin \phi}{R},
$$

to the last term of the exact differential equation of orbit (109) (Figure 1b). Thus, we obtain the approximate differential equation of orbit

$$
\frac{\mathrm{d}^{2} u}{\mathrm{~d} \phi^{2}}+u=3 \frac{G M}{c^{2}} \frac{\sin ^{2} \phi}{R^{2}}=3 \frac{G M}{c^{2} R^{2}}\left(1-\cos ^{2} \phi\right),
$$

with solution

$$
u=\frac{\sin \phi}{R}+3 \frac{G M}{2 c^{2} R^{2}}\left(1+\frac{1}{3} \cos 2 \phi\right) .
$$

Note that the angle $\phi$ is measured from the axis that passes through the center of gravity and is perpendicular to the radius of perihelion $R$ (Figure 1b). For $r \rightarrow+\infty$, we also have

$$
u \rightarrow 0 ; \phi \rightarrow \phi_{\infty} ; \sin \phi_{\infty} \rightarrow \phi_{\infty} ; \cos 2 \phi_{\infty} \rightarrow 1 \text {. }
$$

This gives

$$
\phi_{\infty}=-2 \frac{G M}{c^{2} R}=-\frac{r_{S}}{R^{\prime}}
$$

which is the right-hand deflection. Moreover, there exists the left-hand deflection with

$$
\phi_{\infty l}=\pi+2 \frac{G M}{c^{2} R}=\pi+\frac{r_{S}}{R} .
$$

So, (114) and (115) yield the magnitude of the total deflection of a ray

$$
\Theta=\phi_{\infty l}+\left|\phi_{\infty}\right|-\pi=4 \frac{G M}{c^{2} R}=2 \frac{r_{S}}{R} .
$$


The relevant Newtonian results, for the sake of comparison with the Schwarzschild metric, are [8] (p. 239), [24] (p. 17):

$$
\begin{gathered}
\vec{g}_{N(r)}=-\frac{G M}{r^{2}} \hat{r} ; g_{N(r)}=\frac{G M}{r^{2}} ; \\
L_{N}=\frac{1}{2} m\left(\dot{r}^{2}+r^{2} \dot{\phi}^{2}\right)-\frac{G M m}{r} ; \\
\ddot{r}+\frac{G M}{r^{2}}-r \dot{\phi}^{2}=0 ; J_{N}=m r^{2} \dot{\phi} ; h_{N}=r^{2} \dot{\phi} ;=\frac{\mathrm{d}}{\mathrm{d} t^{\prime}} ;=\frac{\pi}{2} .
\end{gathered}
$$

The Newtonian differential equation of orbit and the corresponding solution are

$$
\begin{aligned}
& \frac{\mathrm{d}^{2} u}{\mathrm{~d} \phi^{2}}+u=\frac{G M}{h_{N}^{2}} ; u=\frac{G M}{h_{N}^{2}}\left(1+e_{N} \cos \phi\right) ; u=\frac{1}{r} ; h_{N}=r^{2} \dot{\phi} ;=\frac{\mathrm{d}}{\mathrm{d} t^{\prime}} ; \\
& e_{N}=\sqrt{1+\frac{2 E_{m N} h_{N}^{2}}{G^{2} M^{2} m}} ; E_{m N}=-\frac{G M m}{2 a_{N}}=-\frac{G M m}{2 R(1+e)^{\prime}} ; \\
& \frac{h_{N}^{2}}{G M}=R_{N}\left(1+e_{N}\right)=a_{N}\left|1-e_{N}^{2}\right|,
\end{aligned}
$$

where $\alpha_{N}$ is the semimajor axis of the Newtonian conic section, which does not rotate $\left(\Delta_{N}=0\right)$. Additionally,

$$
\begin{aligned}
U_{N}=-\frac{G M m}{r} ; V_{N} & =-\frac{G M}{r} ; K_{N}=\frac{1}{2}\left|\vec{\beta}_{P}\right|^{2} m c^{2}=\frac{1}{2} m|\vec{v}|^{2} \\
E_{m N} & =\frac{1}{2} m|\vec{v}|^{2}-\frac{G M}{r} .
\end{aligned}
$$

Finally, the corresponding total deflection of light (which travels with speed c) is [6] (p. 169):

$$
\Theta_{N} \approx \frac{2 G M}{c^{2} R}=\frac{r_{S}}{R} .
$$

The Shapiro Time Delay can be easily obtained from the isotropic form of the Schwarzschild metric [8] (p. 237). Let us consider a light signal passing through the center of gravity $\mathrm{O}$ at (minimum) distance $R \gg r_{S}$, as in Figure 1b. Approximately, the path is given by $x=x, y=R, z=0$. So,

$$
\widetilde{r}^{2}=\widetilde{x}^{2}+\widetilde{R}^{2}=x^{2}+R^{2}=r^{2},
$$

and (30) becomes

$$
0=-\left(\frac{1-\frac{r_{S}}{4 r}}{1+\frac{r_{S}}{4 r}}\right)^{2} c^{2} \mathrm{~d} t^{2}+\left(1+\frac{r_{S}}{4 r}\right)^{4} \mathrm{~d} x^{2} .
$$

The above equation is equivalent to

$$
c^{2} \mathrm{~d} t^{2}=\frac{\left(1+\frac{r_{S}}{4 r}\right)^{6}}{\left(1-\frac{r_{S}}{4 r}\right)^{2}} \mathrm{~d} x^{2}
$$


which is written as

$$
d t=\frac{\left(1+\frac{r_{S}}{4 r}\right)^{3}}{c\left(1-\frac{r_{S}}{4 r}\right)} \mathrm{d} x \approx\left(1+\frac{r_{S}}{r}\right) \frac{\mathrm{d} x}{c}=\left(1+\frac{r_{S}}{\sqrt{x^{2}+R^{2}}}\right) \frac{\mathrm{d} x}{c} .
$$

The integration of (127) from the nearest point of the path to $\mathrm{O}(x=0)$ to the point with $x=X>0$ gives

$$
\Delta t \approx \frac{X}{c}+\frac{r_{S}}{c} \ln \frac{X+\sqrt{X^{2}+R^{2}}}{R} \approx \frac{X}{c}+\frac{r_{S}}{c} \ln \frac{2 X}{R} .
$$

where the last approximation is valid for $X \gg R$. The first term is the result of flat spacetime, while the second expresses the Shapiro time delay. In the case that the signal travels from $X_{1}>0$ on one side to $X_{2}>0$ on the other side, the total Shapiro time delay is

$$
\Delta t_{S} \approx \frac{r_{S}}{c} \ln \frac{\left(X_{1}+\sqrt{X_{1}^{2}+R^{2}}\right)\left(X_{2}+\sqrt{X_{2}^{2}+R^{2}}\right)}{R^{2}} \approx \frac{r_{S}}{c} \ln \frac{4 X_{1} X_{2}}{R^{2}}
$$

The experimental validation of the Shapiro time delay in our Solar system has been done by transponders on the Viking spacecraft in orbit around Mars and on the ground on Mars with an accuracy of $0.1 \%$, in 1979 [25] (p. L220).

\section{SR: Gravitational Field from the Central Scalar Generalized Potential}

4.1. SR Gravitational Central Scalar Generalized Potential, Lagrangian, Equations of Motion, and Correlation to GR Time Dilation

The motion of a particle $P$ (with mass $m$ ) around a center of gravity $O$ (with mass $M$ ) is considered in this section, according to SR. The definition of the Lagrangian of a gravitational system [8] (p. 205) in both the GR and SR [2] (p. 345) gives

$$
L=m \dot{x}^{\mu} g_{\mu v} \dot{x}^{v}=\frac{m \mathrm{~d} S^{2}}{d \tau^{2}}=\frac{-m c^{2} d \tau^{2}}{d \tau^{2}}=-m c^{2} ; \cdot=\frac{\mathrm{d}}{\mathrm{d} \tau} .
$$

In the frame of SR, gravity can only be studied as a field that comes from the $S R$ gravitational potential $\left(V_{S R}, \vec{w}_{S R}\right)$ because the geometry of spacetime has the constant Lorentz metric (9). Thus, more terms are added to the $S R$ Lagrangian of a free particle $P$. This paper examines the case of scalar potential according to the weak EEP (1). So, it is $\vec{w}_{S R}=0$ and the $S R$ Lagrangian in the rest-frame of mass M (RIO O) becomes [2] (p. 351):

$$
L_{S R}=-\frac{1}{\gamma_{\left(\beta_{P}\right)}} m c^{2}-m V_{S R(r, \dot{r}, \dot{\phi})^{\prime}}
$$

where $V_{S R}$ is the $S R$ gravitational central scalar generalized potential. Moreover, central force makes particle $P$ move on the "plane" $\theta=\pi / 2$ and we use polar coordinates for the velocity:

$$
v_{P}^{2}=\dot{r}^{2}+r^{2} \dot{\phi}^{2} ; \gamma_{\left(\beta_{P}\right)}=\frac{1}{\sqrt{1-\frac{\dot{r}^{2}+r^{2} \dot{\phi}^{2}}{c^{2}}}},
$$

which turns the Lagrangian to

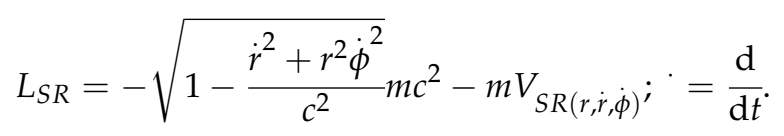


The first integral of motion for the above SR Lagrangian is

$$
E^{*}=\sum_{\mu=1}^{n=2}\left(\frac{\partial L_{S R}}{\partial \dot{x}^{\mu}}\right) \dot{x}^{\mu}-L_{S R} ; \mu=1,2,
$$

which gives

$$
E^{*}=\gamma_{\left(\beta_{P}\right)} m c^{2}+m V_{S R(r, \dot{r}, \dot{\phi})}-m c^{2}\left(\frac{\partial V_{S R}}{\partial \dot{\phi}} \frac{\dot{\phi}}{c^{2}}+\frac{\partial V_{S R}}{\partial \dot{r}} \frac{\dot{r}}{c^{2}}\right) .
$$

The first two terms of the above equation give the quantity

$$
E_{t S R}=\gamma_{\left(\beta_{P}\right)} m c^{2}+m V_{S R(r, \dot{t}, \dot{\phi})^{\prime}}
$$

while the third term is the quantity

$$
E_{d S R}=-m c^{2}\left(\frac{\partial V_{S R}}{\partial \dot{\phi}} \frac{\dot{\phi}}{c^{2}}+\frac{\partial V_{S R}}{\partial \dot{r}} \frac{\dot{r}}{c^{2}}\right) .
$$

Thus, the quantity $E_{\mathrm{tSR}}$ is maintained when

$$
E_{d S R}=\frac{\partial V_{S R}}{\partial \dot{\phi}} \frac{\dot{\phi}}{c^{2}}+\frac{\partial V_{S R}}{\partial \dot{r}} \frac{\dot{r}}{c^{2}}=0 .
$$

For instance, for $V_{\mathrm{SR}}=V_{\mathrm{SR}(r)}$, the $d S R$ energy is null and the $t S R$ energy is maintained [26] (pp. 11-12). Generally, the first integral of motion is the SR total energy

$$
E^{*}=E_{t S R}+E_{d S R}
$$

Moreover, the SR generalized potential energy is defined as

$$
U^{*}=E^{*}-E=m V_{S R(r, \dot{t}, \dot{\phi})}-m c^{2}\left(\frac{\partial V_{S R}}{\partial \dot{\phi}} \frac{\dot{\phi}}{c^{2}}+\frac{\partial V_{S R}}{\partial \dot{r}} \frac{\dot{r}}{c^{2}}\right)
$$

Observe that if the condition (138) is valid (e.g., $V_{\mathrm{SR}}=V_{\mathrm{SR}(r)}$ or the particle is unmoved), then the potential energy is given by the formula:

$$
U=m V_{S R(r, \dot{r}, \dot{\phi})}
$$

It is noted that the coordinate $\phi$ is ignored in the SR Lagrangian (133) and the second integral of motion can be obtained by

$$
J^{*}=\frac{\partial L_{S R}}{\partial \dot{\phi}}
$$

which becomes

$$
J^{*}=m \gamma_{\left(\beta_{P}\right)} r^{2} \dot{\phi}-m \frac{\partial V_{S R}}{\partial \dot{\phi}} .
$$

The first term of the above equation is the $t S R$ angular momentum:

$$
J=m h=m \gamma_{\left(\beta_{P}\right)} r^{2} \dot{\phi} ;=\frac{\mathrm{d}}{\mathrm{d} t^{\prime}}
$$


where $h=J / m$ is the $t S R$ angular momentum per rest mass unit. The second term of (143) is the quantity

$$
J_{d S R}=m h_{d S R}=-m \frac{\partial V_{S R}}{\partial \dot{\phi}}
$$

which is called the $d S R$ angular momentum. Observe that the $t S R$ angular momentum $(J)$ is maintained only when

$$
J_{d S R}=\frac{\partial V_{S R(r, \dot{r}, \dot{\phi})}}{\partial \dot{\phi}}=0
$$

For instance, if $V_{\mathrm{SR}}=V_{\mathrm{SR}(r)}$, the $d S R$ angular momentum is null and the $t S R$ angular momentum $(J)$ is maintained [26] (pp. 11-12). Generally, the second integral of motion is the SR total angular momentum

$$
J^{*}=m h^{*}=J+J_{d S R}=m\left(h+h_{d S R}\right),
$$

where $h^{*}=J^{*} / m$ is the $S R$ total angular momentum per rest mass unit.

Moreover, the Euler-Lagrange equations

$$
\frac{\mathrm{d}}{\mathrm{d} t}\left(\frac{\partial L_{S R}}{\partial \dot{x}^{\mu}}\right)-\frac{\partial L_{S R}}{\partial x^{\mu}}=0 ; \mu=1,2
$$

give the equations of motion:

$$
\begin{gathered}
\frac{\mathrm{d}}{\mathrm{d} t}\left(\gamma_{\left(\beta_{P}\right)} \dot{r}-\frac{\partial V_{S R}}{\partial \dot{r}}\right)-\gamma_{\left(\beta_{P}\right)} r \dot{\phi}^{2}+\frac{\partial V_{S R}}{\partial r}=0 \\
J^{*}=m h^{*}=m \gamma_{\left(\beta_{P}\right)} r^{2} \dot{\phi}-m \frac{\partial V_{S R}}{\partial \dot{\phi}} ; \cdot=\frac{\mathrm{d}}{\mathrm{d} t} .
\end{gathered}
$$

Given all the above, a suitable function for the potential $V_{\mathrm{SR}}$ can be set. Fortunately, GR can assist SR via the EEP in GR: "accelerated motions caused by the gravitational field only (free fall) take place along geodesics of the metric, which corresponds to the particular gravitational field" [2] (p. 248). So, the curved spacetime of GR assumes no force and also the Lorentz $\gamma$-factor is replaced by the GR time dilation $\dot{t}_{G R}$ :

$$
\gamma_{\left(\beta_{P}\right)} \rightarrow \dot{t}_{G R}=\frac{\mathrm{d} t}{\mathrm{~d} \tau_{G R}} .
$$

Moreover, it is

$$
\mathrm{d} S^{2}=-c^{2} \mathrm{~d} \tau_{G R}{ }^{2}
$$

or equivalently,

$$
-c^{2} \frac{\mathrm{d} \tau_{G R^{2}}}{\mathrm{~d} t^{2}}=\frac{\mathrm{d} S^{2}}{\mathrm{~d} t^{2}}
$$

which gives

$$
\dot{t}_{G R}=\frac{\mathrm{d} t}{\mathrm{~d} \tau_{G R}}=\left(\frac{-\mathrm{d} S^{2}}{c^{2} \mathrm{~d} t^{2}}\right)^{-\frac{1}{2}} \geq 1 .
$$

Furthermore, the SR Lagrangian of a free particle P [2] (p. 351) is

$$
L_{S R}=-\frac{1}{\gamma_{\left(\beta_{P}\right)}} m c^{2},
$$


which, with the use of (151), becomes

$$
L_{S R}=-\frac{1}{\dot{t}_{G R}} m c^{2} ; \dot{t}_{G R}=\frac{\mathrm{d} t}{\mathrm{~d} \tau_{G R}} .
$$

Observe that the above SR Lagrangian is not the same as the corresponding GR (32) (because GR refers to spacetime with variable curvature, while SR works in Minkowski spacetime with no curvature), but, as it is shown below, they give exactly the same results. Besides, the combination of (156i) with (131) gives

$$
\frac{1}{\dot{t}_{G R}} m c^{2}=\frac{1}{\gamma_{\left(\beta_{P}\right)}} m c^{2}+m V_{S R(r, \dot{r}, \dot{\phi})} ; \dot{t}_{G R}=\frac{\mathrm{d} t}{\mathrm{~d} \tau_{G R}} .
$$

Thus, we obtain the potential

$$
V_{S R(r, \dot{r}, \dot{\phi})}=c^{2}\left(\frac{1}{\dot{t}_{G R}}-\frac{1}{\gamma_{\left(\beta_{P}\right)}}\right) ; \dot{t}_{G R}=\frac{1}{\frac{V_{S R}}{c^{2}}+\frac{1}{\gamma\left(\beta_{P}\right)}} ; \dot{t}_{G R}=\frac{\mathrm{d} t}{\mathrm{~d} \tau_{G R}}
$$

which contains the GR time dilation $\dot{t}_{G R}=\dot{t}_{G R(r, \dot{r}, \dot{\phi})}$. Of course, the substitution of the potential (158) to (131) yields the SR Lagrangian (156).

Finally, the central scalar generalized potential and the weak EEP (1) give

$$
\vec{F}=m \vec{g} ; \vec{g}=-\frac{\partial V_{S R(r, \dot{r}, \dot{\phi})}}{\partial r} ; g=\frac{\partial V_{S R(r, \dot{r}, \dot{\phi})}}{\partial r},
$$

where $\vec{F} ; \vec{g}$ are the relativistic force and the field strength, respectively [2] (p. 342). The positive value of field strength $(g>0)$ means gravity, while the negative value $(g<0)$ means antigravity.

4.2. SR Gravitational Central Scalar Generalized Potential from the GR Time Dilation of the Schwarzschild Metric: Field Strength, Lagrangian, Equations of Motion, Equation of Trajectory, Precession of Planets' Perihelion, Deflection of Light, and Shapiro Time Delay

The above procedure can be specified by combining the SR with the Schwarzschild metric. The substitution of the time dilation of Schwarzschild metric (50) to (158) gives the SR Schwarzschild Generalized Potential

$$
V_{S R(r, \dot{r}, \dot{\phi})}=c^{2}\left(\left[1-\left(\frac{r_{S}}{r}+\frac{1}{1-\frac{r_{S}}{r} c^{2}}+\frac{\dot{r}^{2} \dot{\phi}^{2}}{c^{2}}\right)\right]^{\frac{1}{2}}-\frac{1}{\gamma_{\left(\beta_{P}\right)}}\right) \cdot=\frac{\mathrm{d}}{\mathrm{d} t} .
$$

The above can be regarded as a modification of the classical Newtonian potential with relativistic effects taken into account and adjustment to the Schwarzschild solution known in GR. Next, the following quantities are calculated:

$$
\begin{aligned}
& \frac{\partial V_{S R}}{\partial \dot{\phi}}=-r^{2} \dot{\phi}\left[1-\left(\frac{r_{S}}{r}+\frac{1}{1-\frac{r_{S}}{r} c^{2}}+\frac{\dot{r}^{2}}{c^{2}}\right) \dot{r}^{2}\right)^{-\frac{1}{2}}+r^{2} \dot{\phi} \gamma_{\left(\beta_{P}\right)} ; \\
& \frac{\partial V_{S R}}{\partial \dot{r}}=-\frac{1}{1-\frac{r_{S}}{r}} \dot{r}\left[1-\left(\frac{r_{S}}{r}+\frac{1}{1-\frac{r_{S}}{r}} \frac{\dot{r}^{2}}{c^{2}}+\frac{r^{2} \dot{\phi}^{2}}{c^{2}}\right)\right]^{-\frac{1}{2}}+\dot{r} \gamma_{\left(\beta_{P}\right)} ;
\end{aligned}
$$




$$
\begin{aligned}
& g= \frac{\partial V_{S R}}{\partial r}= \\
&=c^{2}\left[\begin{array}{l}
\left.\frac{1}{2}\left[\frac{r_{S}}{r^{2}}+\frac{\frac{r_{S}}{r^{2}}}{\left(1-\frac{r_{S}}{r}\right)^{2}} \frac{\dot{r}^{2}}{c^{2}}-\frac{2 r \dot{\phi}^{2}}{c^{2}}\right)\left[1-\left(\frac{r_{S}}{r}+\frac{1}{1-\frac{r_{S}}{r} c^{2}}+\frac{\dot{r}^{2} \dot{\phi}^{2}}{c^{2}}\right)\right]^{-\frac{1}{2}}+\right] \\
+\frac{r \dot{\phi}^{2}}{c^{2}} \gamma_{\left(\beta_{P}\right)}
\end{array}\right]
\end{aligned}
$$

Moreover, the SR Lagrangian (156) becomes

$$
L_{S R}=-m c^{2}\left[1-\left(\frac{r_{S}}{r}+\frac{1}{1-\frac{r_{S}}{r}} \frac{\dot{r}^{2}}{c^{2}}+\frac{r^{2} \dot{\phi}^{2}}{c^{2}}\right)\right]^{\frac{1}{2}} ; \cdot=\frac{\mathrm{d}}{\mathrm{d} t}
$$

which is called the SR Schwarzschild Lagrangian. Furthermore, the substitution of (161) and (162) to (135) implies

$$
\begin{aligned}
& E^{*}=\gamma_{\left(\beta_{P}\right)} m c^{2}+m V_{S R(r, \dot{r}, \dot{\phi})}- \\
& -m c^{2}\left(\begin{array}{c}
-\frac{r^{2} \dot{\phi}^{2}}{c^{2}}\left[1-\left(\frac{r_{S}}{r}+\frac{1}{1-\frac{r_{S}}{r} c^{2}}+\frac{\dot{r}^{2}}{c^{2}}\right)\right]^{2} \dot{\phi}^{2} \\
-\frac{1}{1-\frac{r_{S}}{r} c^{2}}\left[1-\left(\frac{r_{S}}{c^{2}}+\frac{1}{1-\frac{r_{S}}{r}} \gamma_{\left(\beta_{P}\right)}-\frac{\dot{r}^{2}}{c^{2}}+\frac{r^{2} \dot{\phi}^{2}}{c^{2}}\right)\right]^{-\frac{1}{2}}+\frac{\dot{r}^{2}}{c^{2}} \gamma_{\left(\beta_{P}\right)}
\end{array}\right),
\end{aligned}
$$

or equivalently,

$$
E^{*}=\gamma_{\left(\beta_{P}\right)} m c^{2}+m V_{S R(r, \dot{r}, \dot{\phi})}-m c^{2}\left(\begin{array}{l}
{\left[1-\left.\left(\frac{r_{S}}{r}+\frac{1}{1-\frac{r_{S}}{r} c^{2}}+\frac{\dot{r}^{2}}{c^{2}}\right)\right|^{2} \dot{\phi}^{2}\right.} \\
\cdot\left(-\frac{r^{2} \dot{\phi}^{2}}{c^{2}}-\frac{1}{1-\frac{r_{S}}{r} \frac{\dot{r}^{2}}{c^{2}}}\right)+\frac{v^{2}}{c^{2}} \gamma_{\left(\beta_{P}\right)}
\end{array}\right) .
$$

The above equation can be written in the form

$$
E^{*}=\gamma_{\left(\beta_{P}\right)} m c^{2}+m V_{S R(r, \dot{r}, \dot{\phi})}-m c^{2}\left(\begin{array}{l}
{\left[1-\left(\frac{r_{S}}{r}+\frac{1}{1-\frac{r_{S}}{r}}{\frac{\dot{c}^{2}}{2}}^{2} \frac{r^{2} \dot{\phi}^{2}}{c^{2}}\right)\right]^{-\frac{1}{2}} \cdot} \\
\cdot\left(-\frac{r^{2} \dot{\phi}^{2}}{c^{2}}-\frac{1}{1-\frac{r_{S}}{r}} \frac{\dot{r}^{2}}{c^{2}}\right)+\frac{v^{2}}{c^{2}} \frac{\gamma_{\left(\beta_{P}\right)}}{\gamma_{\left(\beta_{P}\right)}}
\end{array}\right)
$$


in order to use the identity

$$
1+\frac{v^{2}}{c^{2}} \gamma_{\left(\beta_{P}\right)}^{2}=\gamma_{\left(\beta_{P}\right)}^{2}
$$

So,

$$
E^{*}=\gamma_{\left(\beta_{P}\right)} m c^{2}+m V_{S R(r, \dot{r}, \dot{\phi})}-m c^{2}\left(\begin{array}{l}
{\left[1-\left(\frac{r_{S}}{r}+\frac{1}{1-\frac{r_{S}}{r}} \frac{\dot{r}^{2}}{c^{2}}+\frac{r^{2} \dot{\phi}^{2}}{c^{2}}\right)\right]^{-\frac{1}{2}}} \\
\cdot\left(-\frac{r^{2} \dot{\phi}^{2}}{c^{2}}-\frac{1}{1-\frac{r_{S}}{r}} \frac{\dot{r}^{2}}{c^{2}}\right)+\frac{\gamma_{\left(\beta_{P}\right)}{ }^{2}-1}{\gamma_{\left(\beta_{P}\right)}}
\end{array}\right),
$$

which is simplified to

$$
E^{*}=m V_{S R(r, \dot{r}, \dot{\phi})}-m c^{2}\left(\begin{array}{l}
{\left[1-\left(\frac{r_{S}}{r}+\frac{1}{1-\frac{r_{S}}{r}} \frac{\dot{r}^{2}}{c^{2}}+\frac{r^{2} \dot{\phi}^{2}}{c^{2}}\right)\right]^{-\frac{1}{2}}} \\
\cdot\left(-\frac{r^{2} \dot{\phi}^{2}}{c^{2}}-\frac{1}{1-\frac{r_{S}}{r}} \frac{\dot{r}^{2}}{c^{2}}\right)-\frac{1}{\gamma_{\left(\beta_{P}\right)}}
\end{array}\right) .
$$

The substitution of (160) to the above, gives

$$
E^{*}=m c^{2}\left(\begin{array}{l}
{\left[1-\left(\frac{r_{S}}{r}+\frac{1}{1-\frac{r_{S}}{r}} \frac{\dot{r}^{2}}{c^{2}}+\frac{r^{2} \dot{\phi}^{2}}{c^{2}}\right)\right]^{\frac{1}{2}}+} \\
+\left[1-\left(\frac{r_{S}}{r}+\frac{1}{1-\frac{r_{S}}{r}} \frac{\dot{r}^{2}}{c^{2}}+\frac{r^{2} \dot{\phi}^{2}}{c^{2}}\right)\right]^{-\frac{1}{2}}\left(\frac{r^{2} \dot{\phi}^{2}}{c^{2}}+\frac{1}{1-\frac{r_{S}}{r}} \frac{\dot{r}^{2}}{c^{2}}\right)
\end{array}\right)
$$

or equivalently,

$$
\begin{aligned}
E^{*}= & m c^{2}\left(1-\left(\frac{r_{S}}{r}+\frac{1}{1-\frac{r_{S}}{r}} \frac{\dot{r}^{2}}{c^{2}}+\frac{r^{2} \dot{\phi}^{2}}{c^{2}}\right)\right)^{-\frac{1}{2}} \cdot \\
& \cdot\left(1-\left(\frac{r_{S}}{r}+\frac{1}{1-\frac{r_{S}}{r}} \frac{\dot{r}^{2}}{c^{2}}+\frac{r^{2} \dot{\phi}^{2}}{c^{2}}\right)+\frac{r^{2} \dot{\phi}^{2}}{c^{2}}+\frac{1}{1-\frac{r_{S}}{r}} \frac{\dot{r}^{2}}{c^{2}}\right) .
\end{aligned}
$$

Hence, the first integral of motion gave the SR total energy

$$
E^{*}=m c^{2}\left[1-\left(\frac{r_{S}}{r}+\frac{1}{1-\frac{r_{S}}{r}} \frac{\dot{r}^{2}}{c^{2}}+\frac{r^{2} \dot{\phi}^{2}}{c^{2}}\right)\right]^{-\frac{1}{2}}\left(1-\frac{r_{S}}{r}\right) \geq 0,
$$


which is exactly equal to the GR total energy (53). Now, considering zero kinetic energy $(K=0)$, when the particle is static $\left(\beta_{P}=0\right)$, we have

$$
E_{\left(\beta_{P}=0\right)}^{*}=E_{\text {rest }}+U \text {, }
$$

where $U$ is the SR gravitational potential energy of a rest particle and

$$
E_{\left(\beta_{P}=0\right)}^{*}=m c^{2} \sqrt{1-\frac{r_{S}}{r}},
$$

is the SR total energy of a rest particle. The substitution of the above equation and (15iii) to (174) gives

$$
U_{(r)}=\left(\sqrt{1-\frac{r_{S}}{r}}-1\right) m c^{2} \leq 0 .
$$

The above combined with (2) gives the SR Schwarzschild Potential of an unmoved particle

$$
V_{(r)}=\left(\sqrt{1-\frac{r_{S}}{r}}-1\right) c^{2} \leq 0 .
$$

This is a central potential with field strength

$$
\vec{g}_{(r)}=-\frac{d V}{d r} \hat{r}=-\frac{G M}{r^{2}}\left(1-\frac{r_{s}}{r}\right)^{-\frac{1}{2}} \hat{r} .
$$

Observe that this result is the same as the corresponding GR formula (60). Furthermore, the SR mechanic energy is

$$
E_{m}=E^{*}-E_{\text {rest }}
$$

So,

$$
E_{m}=\left(\left(1-\frac{r_{S}}{r}\right)\left[1-\left(\frac{r_{S}}{r}+\frac{1}{1-\frac{r_{S}}{r}} \bar{c}^{2}+\frac{\dot{r}^{2} \dot{\phi}^{2}}{c^{2}}\right)\right]^{-\frac{1}{2}}-1\right) m c^{2} ; \theta=\frac{\pi}{2} .
$$

Note that this result is the same as the corresponding GR formula (61). A part of the above energy is the dSR energy. Thus, the combination of (137) with (135) and (169) gives

$$
E_{d S R}=m c^{2}\left(\frac{1}{c^{2}}\left(r^{2} \dot{\phi}^{2}+\frac{1}{1-\frac{r_{S}}{r}} \dot{r}^{2}\right)\left[1-\left(\frac{r_{S}}{r}+\frac{1}{1-\frac{r_{S}}{r}} \dot{r}^{2}+\frac{r^{2} \dot{\phi}^{2}}{c^{2}}\right)\right]^{-\frac{1}{2}}-\frac{\gamma_{\left(\beta_{P}\right)}{ }^{2}-1}{\gamma_{\left(\beta_{P}\right)}}\right) .
$$

Moreover, the lSR mechanic energy is defined as

$$
E_{m l S R}=K+m V_{S R(r, \dot{r}, \dot{\phi})}=E^{*}-E_{\mathrm{rest}}-E_{d S R}
$$

So, (182) with the use of (15i) and (160) gives

$$
E_{m l S R}=m c^{2}\left(\left[1-\left(\frac{r_{S}}{r}+\frac{1}{1-\frac{r_{S}}{r} c^{2}}+\frac{\dot{r}^{2} \dot{\phi}^{2}}{c^{2}}\right)\right]^{-\frac{1}{2}}+\frac{\gamma_{\left(\beta_{P}\right)}{ }^{2}-\gamma_{\left(\beta_{P}\right)}-1}{\gamma_{\left(\beta_{P}\right)}}\right) .
$$


Then, there derives the SR generalized potential energy

$$
U^{*}=E^{*}-E=\left(\left(1-\frac{r_{S}}{r}\right)\left[1-\left(\frac{r_{S}}{r}+\frac{1}{1-\frac{r_{S}}{r}} \frac{\dot{r}^{2}}{c^{2}}+\frac{r^{2} \dot{\phi}^{2}}{c^{2}}\right)\right]^{-\frac{1}{2}}-\gamma_{\left(\beta_{P}\right)}\right) m c^{2} .
$$

Observe that the above formula is not associated with Equation (141), because the condition (138) is not valid for the generalized potential (160). Apart from this, the above result is exactly the same as the corresponding GR formula (62). The case of circular motion is obtained via the substitution of (162) and (163) to the equation of motion (149):

$$
\begin{aligned}
& \frac{\mathrm{d}}{\mathrm{d} t}\left(\frac{1}{1-\frac{r_{S}}{r}} \dot{r}\left[1-\left(\frac{r_{S}}{r}+\frac{1}{1-\frac{r_{S}}{r}} \dot{r}^{2}+\frac{r^{2} \dot{\phi}^{2}}{c^{2}}\right)\right]^{-\frac{1}{2}}\right)+ \\
& +\frac{c^{2}}{2}\left(\frac{r_{S}}{r^{2}}+\frac{\frac{r_{S}}{r^{2}}}{\left(1-\frac{r_{S}}{r}\right)^{2}}{\dot{r^{2}}}^{2}-\frac{2 r \dot{\phi}^{2}}{c^{2}}\right)\left[1-\left(\frac{r_{S}}{r}+\frac{1}{1-\frac{r_{S}}{r}} c^{2}+\frac{\dot{r}^{2} \dot{\phi}^{2}}{c^{2}}\right)\right]^{-\frac{1}{2}}=0 .
\end{aligned}
$$

For $r=R=$ constant, the equality (186) holds:

$$
\frac{r_{S}}{R^{2}}-\frac{2 R \dot{\phi}^{2}}{c^{2}}=0 .
$$

This gives the UCM, with the same angular velocity and the same centripetal acceleration in classical mechanics and in SR, which are exactly the same as in the GR (43):

$$
\omega=\dot{\phi}=\frac{\mathrm{d} \phi}{\mathrm{d} t}=\sqrt{\frac{G M}{R^{3}}} ; a=\frac{v^{2}}{R}=\omega^{2} R=\frac{G M}{R^{2}}=g_{N},
$$

As shown below, the orbit of motion derives via a similar way to the original Schwarzschild space [8] (pp. 238-45). The substitution of (161) to (150) gives

$$
h^{*}=r^{2} \dot{\phi}\left[1-\left(\frac{r_{S}}{r}+\frac{1}{1-\frac{r_{S}}{r}} \frac{\dot{r}^{2}}{c^{2}}+\frac{r^{2} \dot{\phi}^{2}}{c^{2}}\right)\right]^{-\frac{1}{2}} ; \cdot=\frac{\mathrm{d}}{\mathrm{d} t} .
$$

Additionally, (173) implies

$$
\left[1-\left(\frac{r_{S}}{r}+\frac{1}{1-\frac{r_{S}}{r}} c^{2}+\frac{\dot{r}^{2} \dot{\phi}^{2}}{c^{2}}\right)\right]^{-\frac{1}{2}}=\frac{E^{*}}{m c^{2}\left(1-\frac{r_{S}}{r}\right)} .
$$

Equation (188), with the use of the above (189), becomes

$$
\dot{\phi}=\frac{m c^{2} h^{*}\left(1-\frac{r_{S}}{r}\right)}{E^{*} r^{2}} ; \cdot=\frac{\mathrm{d}}{\mathrm{d} t} .
$$


Moreover, (189) is written as

$$
\left[1-\left(\frac{r_{S}}{r}+\frac{1}{1-\frac{r_{S}}{r}} \frac{1}{c^{2}}\left(\frac{\mathrm{d} r}{\mathrm{~d} \phi}\right)^{2} \dot{\phi}^{2}+\frac{r^{2} \dot{\phi}^{2}}{c^{2}}\right)\right]^{\frac{1}{2}}=\frac{m c^{2}\left(1-\frac{r_{S}}{r}\right)}{E^{*}}
$$

which, combined with (190) gives

$$
\left[1-\left(\frac{r_{S}}{r}+\left(\frac{\mathrm{d} r}{\mathrm{~d} \phi}\right)^{2} \frac{m^{2} c^{2} h^{* 2}\left(1-\frac{r_{S}}{r}\right)}{E^{* 2} r^{4}}+\frac{m^{2} c^{2} h^{* 2}\left(1-\frac{r_{S}}{r}\right)^{2}}{E^{* 2} r^{2}}\right)\right]^{\frac{1}{2}}=\frac{m c^{2}\left(1-\frac{r_{S}}{r}\right)}{E^{*}} .
$$

The following

$$
u=\frac{1}{r} ; r=\frac{1}{u^{\prime}} ; \frac{\mathrm{d} r}{\mathrm{~d} \phi}=-\frac{1}{u^{2}} \frac{\mathrm{d} u}{\mathrm{~d} \phi}=-r^{2} \frac{\mathrm{d} u}{\mathrm{~d} \phi^{\prime}}
$$

transform (192) to

$$
\begin{aligned}
& {\left[1-\left(r_{S} u+\left(\frac{\mathrm{d} u}{\mathrm{~d} \phi}\right)^{2} \frac{m^{2} c^{2} h^{* 2}\left(1-r_{S} u\right)}{E^{* 2}}+\frac{m^{2} c^{2} h^{* 2}\left(1-r_{S} u\right)^{2} u^{2}}{E^{* 2}}\right)\right]^{\frac{1}{2}}=} \\
& =\frac{m c^{2}\left(1-r_{S} u\right)}{E^{*}} .
\end{aligned}
$$

Thus, the above equation gives

$$
1-r_{S} u-\left(\frac{\mathrm{d} u}{\mathrm{~d} \phi}\right)^{2} \frac{m^{2} c^{2} h^{* 2}\left(1-r_{S} u\right)}{E^{* 2}}-\frac{m^{2} c^{2} h^{* 2}\left(1-r_{S} u\right)^{2} u^{2}}{E^{* 2}}=\frac{m^{2} c^{4}\left(1-r_{S} u\right)^{2}}{E^{* 2}}
$$

which is equivalent to

$$
1-\left(\frac{\mathrm{d} u}{\mathrm{~d} \phi}\right)^{2} \frac{m^{2} c^{2} h^{* 2}}{E^{* 2}}-\frac{m^{2} c^{2} h^{* 2}\left(1-r_{S} u\right) u^{2}}{E^{* 2}}=\frac{m^{2} c^{4}\left(1-r_{S} u\right)}{E^{* 2}} .
$$

Equation (196) becomes

$$
\left(\frac{\mathrm{d} u}{\mathrm{~d} \phi}\right)^{2}+\left(1-r_{S} u\right) u^{2}=-\frac{c^{2}\left(1-r_{S} u\right)}{h^{* 2}}+\frac{E^{* 2}}{m^{2} c^{2} h^{* 2}}
$$

which is differentiated with respect to $\phi$ and gives

$$
2 \frac{\mathrm{d} u \mathrm{~d}^{2} u}{\mathrm{~d} \phi} \frac{\mathrm{d} \phi^{2}}{\mathrm{~d}}+2 u \frac{\mathrm{d} u}{\mathrm{~d} \phi}-3 r_{S} u^{2} \frac{\mathrm{d} u}{\mathrm{~d} \phi}=\frac{c^{2} r_{S}}{h^{* 2}} \frac{\mathrm{d} u}{\mathrm{~d} \phi^{\prime}}
$$

or equivalently,

$$
\frac{\mathrm{d}^{2} u}{\mathrm{~d} \phi^{2}}+u-\frac{3}{2} r_{S} u^{2}=\frac{c^{2} r_{S}}{2 h^{* 2}}
$$

Thus, the equation of trajectory for SR Schwarzschild Generalized Potential (160) is obtained:

$$
\frac{\mathrm{d}^{2} u}{\mathrm{~d} \phi^{2}}+u=\frac{G M}{h^{* 2}}+3 \frac{G M}{c^{2}} u^{2} ; u=\frac{1}{r^{\prime}}
$$


where, from $(188), h^{*}$ is

$$
h^{*}=h_{N}\left[1-\left(\frac{r_{S}}{r}+\frac{1}{1-\frac{r_{S}}{r}} \frac{\dot{r}^{2}}{c^{2}}+\frac{r^{2} \dot{\phi}^{2}}{c^{2}}\right)\right]^{-\frac{1}{2}} ; h_{N}=r^{2} \dot{\phi} ;=\frac{\mathrm{d}}{\mathrm{d} t} .
$$

Observe that the above equation of trajectory and the angular momentum are exactly the same as the corresponding GR (63) and (76). So, it is easy to obtain the SR results, by just replacing

$$
h_{G R} \rightarrow h^{*} ; E_{G R} \rightarrow E^{*}
$$

to the Schwarzschild metric results in Section 3.3. Thus, the same precession of the ellipse that rotates about one of its foci (91) and the same magnitude of the total deflection of light (116) are obtained again.

The deeper cause for the coincidence of the SR and GR approaches is the fact that even though the SR Lagrangian (164) and the GR Lagrangian (32) are different, their Euler-Lagrange equations of motion are the same. Indeed, the first GR Euler-Lagrange equation (34) and the GR time dilation (50) yield the GR total energy $\left(E_{\mathrm{GR}}\right)$ (53). This is exactly the same as the $S R$ total energy $\left(E^{*}\right)(173)$, which comes from the SR first integral of motion (134). Additionally, the second SR Euler-Lagrange equation (149) combined with the GR time dilation (50) and formulas (162)-(163) give the second GR Euler-Lagrange equation (35). Furthermore, the third $S R$ Euler-Lagrange equation (150) combined with the GR time dilation (50) and formula (161) yield the third GR Euler-Lagrange equation (36).

In the case of UCM, the SR approach gives

$$
\begin{gathered}
\omega=\dot{\phi}=\frac{\mathrm{d} \phi}{\mathrm{d} t}=\sqrt{\frac{G M}{R^{3}}} ; a=\frac{v^{2}}{R}=\omega^{2} R=\frac{G M}{R^{2}}=g_{N} \\
v=\sqrt{\frac{G M}{R}} ; g=\gamma_{\left(\beta_{P}\right)} \frac{G M}{R^{2}}=\frac{1}{\sqrt{1-\frac{r_{S}}{2 R}}} \frac{G M}{R^{2}} ; \\
h^{*}=\sqrt{G M R}\left(1-\frac{3}{c^{2}} \frac{G M}{R}\right)^{-\frac{1}{2}} ; E_{m}=\left(\left(1-\frac{r_{S}}{r}\right)\left(1-\frac{3}{c^{2}} \frac{G M}{R}\right)^{-\frac{1}{2}}-1\right) m c^{2} .
\end{gathered}
$$

The gravitational field on a momentarily unmoved particle is studied next. Equation (163) becomes

$$
g=\frac{\partial V_{S R}}{\partial r}=\frac{G M}{r^{2}}\left(1-\frac{r_{S}}{r}\right)^{-\frac{1}{2}} .
$$

While (205) is the same as the corresponding Schwarzschild metric (25), it is much different than the corresponding UCM (203iv). The initial acceleration is calculated as follows: For $\dot{\phi}=0$, (42) becomes

$$
2 \ddot{r}+\left(1-\frac{r_{S}}{r}\right) \frac{r_{S}}{r^{2}} c^{2}=0,
$$

which, combined with (4), implies

$$
a_{r}=\ddot{r}=-\frac{G M}{r^{2}}\left(1-\frac{r_{S}}{r}\right) .
$$

It becomes evident that the initial acceleration of a momentarily unmoved particle is different than the corresponding field strength (205) and (25). Moreover, observe that the acceleration of 
an unmoved particle on the Schwarzschild radius $\left(r=r_{\mathrm{S}}\right)$ is null, while the corresponding field strength is infinite!

In the case of the planet Mercury, it is $\alpha=0.38709893 \mathrm{AU}, e=0.20563069$, and $T=87.968$ days [27]. The values: $\mathrm{AU}=1.4959787066 \times 10^{11} \mathrm{~m}, \mathrm{G}=6.67428(67) \times$ $10^{-11} \mathrm{~m}^{3} \mathrm{~kg}^{-1} \mathrm{~s}^{-2}, c=299,792,458 \mathrm{~ms}^{-1}$ (exact) [28] (pp. 1-1, 1-20, 14-2), and $M=1,988,500 \times 10^{24} \mathrm{~kg}[29]$ give

$$
\frac{r_{S}}{a\left(1-e^{2}\right)}=\frac{2 G M}{c^{2} a\left(1-e^{2}\right)}=5.32518(53) \times 10^{-8} \ll 1 .
$$

In the case of the Earth where $\alpha=1.00000011 \mathrm{AU}, e=0.01671022$, and $T=365.242$ days [30], the calculation gives

$$
\frac{r_{S}}{a\left(1-e^{2}\right)}=\frac{2 G M}{c^{2} a\left(1-e^{2}\right)}=1.97476(20) \times 10^{-8} \ll 1 .
$$

Now, with the above values, the combination of (93) and (91) gives the results in Table 1. Observe that both SR and GR give the same precessions.

Table 1. Angular velocity of perihelion rotation ("precession") for Mercury and Earth according to the gravitational field of the SR Schwarzschild Generalized Potential $\left(\Omega_{\mathrm{SR}}\right)$ and the Schwarzschild metric

\begin{tabular}{|c|c|c|c|c|c|}
\hline Mercury & & & Earth & & \\
\hline$\Omega_{\mathrm{SR}} /{ }^{\prime \prime} \mathrm{cy}^{-1}$ & $\Omega_{\mathrm{GR}} /{ }^{\prime \prime} \mathrm{cy}^{-1}$ & $\Delta \Omega_{\mathrm{SRr}}(\%)$ & $\Omega_{\mathrm{SR}} /{ }^{\prime \prime} \mathrm{cy}^{-1}$ & $\Omega_{\mathrm{GR}} /{ }^{\prime \prime} \mathrm{cy}^{-1}$ & $\Delta \Omega_{\mathrm{SRr}}(\%)$ \\
\hline $42.9820(43)^{(1)}$ & $42.9820(43)^{(1)}$ & 0 & $3.83893(38)^{(1)}$ & $3.83893(38)^{(1)}$ & 0 \\
\hline
\end{tabular}
$\left(\Omega_{\mathrm{GR}}\right)$, respectively. $\Delta \Omega_{\mathrm{SRr}}(\%)$ is the percentile relative change.

${ }^{1}$ data from $[27,28]$ (pp. 1-1, 1-20, 14-2), [29,30].

\subsection{SR Gravitational Central Scalar Generalized Potential from the Time Dilation of Teleparallel Gravity}

The above procedure can also apply when combining the SR with Lorentz Gauge Theory [9-14], Teleparallel Gravity [15-21], etc., with the use of the corresponding time dilation. Furthermore, the gravitational Poincaré Gauge Theory formulated on Weitzenböck spacetime $\left(\mathrm{T}^{4}\right)[9]$ (pp. 57, 68) is called Teleparallel Gravity. This is characterized by vanishing the curvature tensor (absolute parallelism) and the torsion tensor, which comes from four parallel vector fields. Albert Einstein in 1928 first gave its original form [15]. So, this theory is called new general relativity [21] (p. 3524). The corresponding isotropic metric by a static, spherical body (assuming that the spin of constituent particles of a body can be completely neglected) [21] (pp. 3533-3537) is

$$
\begin{aligned}
\mathrm{d} S^{2} & =-c^{2} \mathrm{~d} \tau_{G R}{ }^{2}=-\left(1-\frac{r_{S}}{2 p \widetilde{r}}\right)^{p}\left(1+\frac{r_{S}}{2 q \widetilde{r}}\right)^{-q} c^{2} \mathrm{~d} t^{2}+ \\
& +\left(1-\frac{r_{S}}{2 p \widetilde{r}}\right)^{2-p}\left(1+\frac{r_{S}}{2 q \widetilde{r}}\right)^{2+q}\left(\mathrm{~d} \widetilde{r}^{2}+\widetilde{r}^{2} \mathrm{~d} \theta^{2}+\widetilde{r}^{2} \sin ^{2} \theta \mathrm{d} \phi^{2}\right)
\end{aligned}
$$

where

$$
\begin{gathered}
p=\frac{2}{1-5 \varepsilon}[\sqrt{(1-\varepsilon)(1-4 \varepsilon)}-2 \varepsilon] ; q=\frac{2}{1-5 \varepsilon}[\sqrt{(1-\varepsilon)(1-4 \varepsilon)}+2 \varepsilon] ; \\
\varepsilon=\frac{k\left(c_{1}+c_{2}\right)}{1+k\left(c_{1}+4 c_{2}\right)} ; c_{1}=a_{1}+\frac{1}{3 k^{\prime}} c_{2}=a_{2}-\frac{1}{3 k^{\prime}} ; k=\frac{8 \pi G}{c^{4}}
\end{gathered}
$$


and $c_{1} ; c_{2} ; a_{1} ; a_{2}$ are constant parameters that come from the corresponding gravitational action $I_{G}$. Besides, we have

$$
\begin{aligned}
\varepsilon & =\frac{k\left(c_{1}+c_{2}\right)}{1+k\left(c_{1}+4 c_{2}\right)}=\frac{1}{\frac{1+3 k c_{2}}{k\left(c_{1}+c_{2}\right)}+1}= \\
= & \frac{1}{\frac{1+3 k\left(a_{2}-\frac{1}{3 k}\right)}{k\left(a_{1}+a_{2}\right)}+1}=\frac{1}{\frac{3 a_{2}}{a_{1}+a_{2}}+1}=\frac{1}{1+\frac{3}{1+\frac{a_{1}}{a_{2}}}}
\end{aligned}
$$

Since the parameters $p$ and $q$ are real numbers, we have

$$
-\infty<\varepsilon \leq \frac{1}{4} \text { for }-4<\frac{a_{1}}{a_{2}} \leq 0,
$$

or

$$
\varepsilon \geq 1 \text { for } \frac{a_{1}}{a_{2}}<-4 \text {. }
$$

Observe that for $c_{1}=c_{2}=0, \varepsilon$ is equal to 0 . Thus, it is $p=q=2$ and metric (210) becomes the (Riemannian) isotropic form of the Schwarzschild metric (27) [21] (p. 3537). This happens because the field equations in Weitzenbock spacetime are reduced to Einstein's field equations under two conditions: (i) the axial-vector part of the torsion tensor vanishes identically; and (ii) effects due to the intrinsic spin $-1 / 2$ of the fundamental particles can be neglected [21] (p. 3539). In this case, where $\varepsilon=0(p=q=2)$, the corresponding SR gravitational generalized potential is exactly the SR Schwarzschild Generalized Potential given by formula (160). In any other case $\varepsilon \neq 0(p \neq 2, q \neq 2)$, we have to calculate the corresponding time dilation in Weitzenbock spacetime. This can be done with the use of isotropic metric (210), but it is better to transform the metric to

$$
\mathrm{d} S^{2}=-c^{2} \mathrm{~d} \tau_{G R}{ }^{2}=-f_{(r)} c^{2} \mathrm{~d} t^{2}+g_{(r)} \mathrm{d} r^{2}+r^{2} \mathrm{~d} \theta^{2}+r^{2} \sin ^{2} \theta \mathrm{d} \phi^{2},
$$

in order to compare it with the original Schwarzschild solution of Einstein's field equations in a vacuum. The coefficients of $\mathrm{d} \theta^{2}$ in metrics (210) and (216) imply

$$
r=\widetilde{r}\left(1-\frac{r_{S}}{2 p \widetilde{r}}\right)^{1-\frac{p}{2}}\left(1+\frac{r_{S}}{2 q \widetilde{r}}\right)^{1+\frac{q}{2}}
$$

and (210) becomes

$$
\begin{aligned}
\mathrm{d} S^{2} & =-c^{2} \mathrm{~d} \tau_{G R}{ }^{2}=-\left(1-\frac{r_{S}}{2 p \widetilde{r}}\right)^{p}\left(1+\frac{r_{S}}{2 q \widetilde{r}}\right)^{-q} c^{2} \mathrm{~d} t^{2}+ \\
& +\left(1-\frac{r_{S}}{2 p \widetilde{r}}\right)^{2-p}\left(1+\frac{r_{S}}{2 q \widetilde{r}}\right)^{2+q} \mathrm{~d} \widetilde{r}^{2}+r^{2} \mathrm{~d} \theta^{2}+r^{2} \sin ^{2} \theta \mathrm{d} \phi^{2}
\end{aligned}
$$

Unfortunately, we cannot obtain the accurate function $\widetilde{r}=\widetilde{r}(r)$. So, we have to apply the following approximation in (217) (a first-order Taylor theorem around $p=q=2$ for variables $\mathrm{p}, \mathrm{q}$ and then a first-order Taylor theorem for $\widetilde{r} \gg r_{S}$ ):

$$
r \cong \widetilde{r}\left(1+\frac{r_{S}}{4 \widetilde{r}}\right)^{2}\left(1+\frac{r_{S}}{4 \widetilde{r}} \frac{p-q}{2}\right)=\widetilde{r}\left(1+\frac{r_{S}}{4 \widetilde{r}}\right)^{2}\left(1+\frac{r_{S}}{4 \widetilde{r}} \frac{-4 \varepsilon}{1-5 \varepsilon}\right)
$$


The experimental value of $\varepsilon$ (solar system observations of light gravitational deflection and precession of Mercury's perihelion) is [21] (p. 3538):

$$
\varepsilon=-0.004 \pm 0.004
$$

Thus, (218) becomes

$$
r \cong \widetilde{r}\left(1+\frac{r_{S}}{4 \widetilde{r}}\right)^{2}\left(1+0.0157 \frac{r_{S}}{4 \widetilde{r}}\right)
$$

and we obtain the approximate function

$$
\begin{aligned}
& r \cong \widetilde{r}\left(1+\frac{r_{S}}{4 \widetilde{r}}\right)^{2}\left(1+0.0157 \frac{r_{S}}{4 \widetilde{r}}\right) \cong \widetilde{r}\left(1+\frac{r_{S}}{4 \widetilde{r}}\right)^{2} ; \\
& \widetilde{r} \cong \frac{r_{S}}{4}\left(\frac{2 r}{r_{S}}\left(1+\sqrt{1-\frac{r_{S}}{r}}\right)-1\right) \cong r-\frac{r_{S}}{2} .
\end{aligned}
$$

We observe that we have approximately obtained the original Schwarzschild result (26). The comparison of metrics (22) and (27) also implies

$$
\left(\frac{1-\frac{r_{S}}{4 \widetilde{r}}}{1+\frac{r_{S}}{4 \widetilde{r}}}\right)^{2}=1-\frac{r_{S}}{r} ;\left(1+\frac{r_{S}}{4 \widetilde{r}}\right)^{4} \mathrm{~d} \widetilde{r}^{2}=\frac{1}{1-\frac{r_{S}}{r}} \mathrm{~d} r^{2},
$$

which gives

$$
\mathrm{d} \widetilde{r}^{2}=\frac{1}{\left(1-\frac{r_{S}}{r}\right)\left(1+\frac{r_{S}}{4 \widetilde{r}}\right)^{4}} \mathrm{~d} r^{2} .
$$

The substitution of (224) to metric (218) gives

$$
\begin{aligned}
& \mathrm{d} S^{2}=-c^{2} \mathrm{~d} \tau_{G R}{ }^{2}=-\left(1-\frac{r_{S}}{2 p \widetilde{r}}\right)^{p}\left(1+\frac{r_{S}}{2 q \widetilde{r}}\right)^{-q} c^{2} \mathrm{~d} t^{2}+ \\
& +\left(1-\frac{r_{S}}{2 p \widetilde{r}}\right)^{2-p}\left(1+\frac{r_{S}}{2 q \widetilde{r}}\right)^{2+q} \frac{1}{\left(1-\frac{r_{S}}{r}\right)\left(1+\frac{r_{S}}{4 \widetilde{r}}\right)^{4}} \mathrm{~d} r^{2}+ \\
& \quad+r^{2} \mathrm{~d} \theta^{2}+r^{2} \sin ^{2} \theta \mathrm{d} \phi^{2},
\end{aligned}
$$

which is the metric according to the Teleparallel Gravity that corresponds to the original Schwarzschild metric. Once again, the approximation (a first-order Taylor theorem around $p=q=2$ for variables $p, q$ and then a first-order Taylor theorem for $\tilde{r} \gg r_{S}$ ) gives:

$$
\begin{aligned}
f_{(r)} & =\left(1-\frac{r_{S}}{2 p \widetilde{r}}\right)^{p}\left(1+\frac{r_{S}}{2 q \widetilde{r}}\right)^{-q} \cong \\
& \cong\left(1+\frac{r_{S}}{4 \widetilde{r}}\right)^{2}\left(1-\frac{r_{S}}{4 \widetilde{r}}\right)^{-2}+0(p-2)+0(q-2)=1-\frac{r_{S}}{r} .
\end{aligned}
$$


Thus, the coefficient of time in Teleparallel Gravity is approximately the same as the corresponding Schwarzschild metric. Moreover, the same approximation gives:

$$
\begin{aligned}
g_{(r)} & =\left(1-\frac{r_{S}}{2 p \widetilde{r}}\right)^{2-p}\left(1+\frac{r_{S}}{2 q \widetilde{r}}\right)^{2+q} \frac{1}{\left(1-\frac{r_{S}}{r}\right)\left(1+\frac{r_{S}}{4 \widetilde{r}}\right)^{4}} \cong \\
& \cong \frac{1}{\left(1-\frac{r_{S}}{r}\right)\left(1+\frac{r_{S}}{4 \widetilde{r}}\right)^{4}}\left[\left(1+\frac{r_{S}}{4 \widetilde{r}}\right)^{4}+\frac{r_{S}}{4 \widetilde{r}}(p-2)-\frac{r_{S}}{4 \widetilde{r}}\left(1+\frac{r_{S}}{4 \widetilde{r}}\right)^{4}(q-2)\right]
\end{aligned}
$$

The above is rewritten as

$$
g_{(r)} \cong \frac{\left(1+\frac{r_{S}}{4 \widetilde{r}}\right)^{4}}{\left(1-\frac{r_{S}}{r}\right)\left(1+\frac{r_{S}}{4 \widetilde{r}}\right)^{4}}\left[1+\frac{r_{S}}{4 \widetilde{r}}\left(1+\frac{r_{S}}{4 \widetilde{r}}\right)^{-4}(p-2)-\frac{r_{S}}{4 \widetilde{r}}(q-2)\right]
$$

which becomes

$$
g_{(r)} \cong \frac{1}{1-\frac{r_{S}}{r}}\left[1+\frac{r_{S}}{4 \widetilde{r}}\left(1-\frac{r_{S}}{4 \widetilde{r}}\right)(p-2)-\frac{r_{S}}{4 \widetilde{r}}(q-2)\right]
$$

with the use of the approximation (the first-order Taylor theorem for $\widetilde{r} \gg r_{S}$ ). Furthermore, we have

$$
g_{(r)} \cong \frac{1}{1-\frac{r_{S}}{r}}\left[1+\frac{r_{S}}{4 \widetilde{r}}(p-q)-\frac{r_{S}^{2}}{16 \widetilde{r}^{2}}(q-2)\right],
$$

where the last term is extremely small. Thus, we obtain

$$
\begin{aligned}
g_{(r)} & \cong \frac{1}{1-\frac{r_{S}}{r}}\left[1+\frac{r_{S}}{4 \widetilde{r}}(p-q)\right]= \\
& =\frac{1}{1-\frac{r_{S}}{r}}\left(1+\frac{r_{S}}{4 \widetilde{r}} \frac{-8 \varepsilon}{1-5 \varepsilon}\right) \cong \frac{1}{1-\frac{r_{S}}{r}}\left(1-\frac{8 \varepsilon}{1-5 \varepsilon} \frac{r_{S}}{4 r}\right),
\end{aligned}
$$

So, the coefficient of $\mathrm{d} r^{2}$ is a little different from the corresponding Schwarzschild metric. Finally, the metric by a static, spherical body (assuming that the spin of constituent particles of a body can be completely neglected) according to the Teleparallel Gravity is approximately:

$$
\begin{aligned}
\mathrm{d} S^{2} & =-c^{2} \mathrm{~d} \tau_{G R}{ }^{2}=-\left(1-\frac{r_{S}}{r}\right) c^{2} \mathrm{~d} t^{2}+ \\
& +\frac{1}{1-\frac{r_{S}}{r}}\left(1-\frac{8 \varepsilon}{1-5 \varepsilon} \frac{r_{S}}{4 r}\right) \mathrm{d} r^{2}+r^{2} \mathrm{~d} \theta^{2}+r^{2} \sin ^{2} \theta \mathrm{d} \phi^{2},
\end{aligned}
$$

We observe that if $\varepsilon \rightarrow 0(p=q \rightarrow 2)$, the above metric becomes the (Riemannian) Schwarzschild metric (22) and the corresponding SR gravitational generalized potential is the SR Schwarzschild Generalized Potential given by formula (160). In any other case: $\varepsilon \neq 0$ 
$p \neq 2, q \neq 2$ ), we have to calculate the corresponding time dilation in Weitzenbock spacetime.

Thus, we divide the above equation with $-c^{2} \mathrm{~d} t^{2}$ and we have for $\theta=\frac{\pi}{2}$ :

$$
\left(\frac{\mathrm{d} \tau_{G R}}{\mathrm{~d} t}\right)^{2}=\left(1-\frac{r_{S}}{r}\right)-\frac{1}{1-\frac{r_{S}}{r}}\left(1-\frac{8 \varepsilon}{1-5 \varepsilon} \frac{r_{S}}{4 r}\right)\left(\frac{d r}{\mathrm{~d} t}\right)^{2} \frac{1}{c^{2}}-r^{2}\left(\frac{\mathrm{d} \phi}{\mathrm{d} t}\right)^{2} \frac{1}{c^{2}} .
$$

This gives the connecting relation of the proper time of Teleparallel Gravity with the time of RIO O (the Teleparallel Gravity GR time dilation with respect to RIO O)

$$
\frac{\mathrm{d} t}{\mathrm{~d} \tau_{G R}}=\left[1-\left(\frac{r_{S}}{r}+\left(1-\frac{8 \varepsilon}{1-5 \varepsilon} \frac{r_{S}}{r r}\right) \frac{1}{1-\frac{r_{S}}{r} c^{2}}+\frac{\dot{r}^{2} \dot{\phi}^{2}}{c^{2}}\right)\right]^{-\frac{1}{2}} \geq 1 ;=\frac{\mathrm{d}}{\mathrm{d} t} .
$$

The substitution of the above time dilation to (158) gives the corresponding SR Teleparallel Gravity Generalized Potential by a static, spherical body (assuming that the spin of constituent particles of a static spherical body is completely neglected):

$$
\begin{aligned}
V_{S R(r, \dot{r}, \dot{\phi})}=c^{2}([1- & \left.\left(\frac{r_{S}}{r}+\left(1-\frac{8 \varepsilon}{1-5 \varepsilon} \frac{r_{S}}{4 r}\right) \frac{1}{1-\frac{r_{S}}{r} c^{2}} \cdot+\frac{\dot{r}^{2}}{c^{2}}\right)\right]^{2}- \\
& \left.-\frac{1}{\gamma_{\left(\beta_{P}\right)}}\right) ; \cdot=\frac{\mathrm{d}}{\mathrm{d} t} .
\end{aligned}
$$

We observe that the GR Schwarzschild time dilation (50) and the SR Schwarzschild Generalized Potential (160) can be regarded as the limit of corresponding quantities of Teleparallel Gravity for $\varepsilon \rightarrow 0(p=q \rightarrow 2)$.

\section{Gravitational Red Shift}

In the following, the first part presents the Gravitational Red Shift according to GR. Let us consider two consecutive wave fronts passing from point $A$, which is located at distance $r_{0}$ from the center of gravity $\mathrm{O}$. So, there are two events: $A\left(t_{1}\right), A\left(t_{2}\right)$. Then, the Schwarzschild metric (22) gives

$$
d S_{A}^{2}=-c^{2} d \tau_{A}^{2}=-\left(1-\frac{r_{S}}{r_{0}}\right) c^{2} d t_{A}^{2} .
$$

The square root and integration of the above (for one period of the wave/vibration) gives

$$
T_{\left(r_{0}\right)}^{*}=\sqrt{1-\frac{r_{S}}{r_{0}}} T_{\left(r_{0}\right)}
$$

where $T_{\left(r_{0}\right)}^{*} T_{\left(r_{0}\right)}$ are the periods of the vibration at point $A$ for the unmoved observer located at $A$ (local observer $A$ ) and the RIO O, respectively. Moreover, (237) implies

$$
f_{\left(r_{0}\right)}^{*}=\frac{1}{\sqrt{1-\frac{r_{S}}{r_{0}}}} f_{\left(r_{0}\right)^{\prime}}
$$


where $f_{\left(r_{0}\right)}^{*}, f_{\left(r_{0}\right)}$ are the frequencies of the vibration at point $A$ for the local observer $A$ and the RIO O, respectively. Thus, the corresponding GR total energies of a photon can be related as follows:

$$
E_{G R\left(r_{0}\right)}^{*}=E_{G R}\left(1-\frac{r_{S}}{r_{0}}\right)^{-\frac{1}{2}}
$$

where $E_{G R\left(r_{0}\right)}^{*} ; E_{\mathrm{GR}}$ are the energies of a photon measured by local observer $A$ and RIO $\mathrm{O}$, respectively, $E_{G R\left(r_{0}\right)}^{*}=h f_{\left(r_{0}\right)}^{*}, E_{G R}=h f_{\left(r_{0}\right)}$ and $\mathrm{h}$ is the Plank constant. Evidently, Equation (57) is obtained once again. Moreover, the definition of the $z$-factor of Gravitational Red Shift is

$$
z=\frac{\lambda_{O}-\lambda_{E L}}{\lambda_{E L}}=\frac{\lambda_{O}}{\lambda_{E L}}-1=\frac{\frac{c_{E}}{f_{O}}}{\frac{c_{E}}{f_{E L}}}-1=\frac{f_{E L}}{f_{O}}-1,
$$

where $\lambda_{\mathrm{O}}$ is the observed wavelength of radiation, which is produced at distance $r_{0}$ from the center of gravity and $\lambda_{\mathrm{EL}}$ is the wavelength of the corresponding radiation that is produced in an Earth laboratory (both of them are measured by unmoved observers on the Earth, where the speed of light is $\left.c_{\mathrm{E}}\right)$.

According to the literature (e.g., [8] (p. 188)), the periods/frequencies of the wave/vibration are considered to be steady for the RIO O:

$$
T_{\left(r_{0}\right)}=t_{2}-t_{1}=T_{\infty} ; f_{\left(r_{0}\right)}=f_{\infty} .
$$

This consideration is valid only approximately, because the speed of a photon is variable around the center of gravity $\mathrm{O}$ (see formulas (71i) and (73)). So, the corresponding electromagnetic wave has a variable period as well and this will be shown in the non-mainstream SR approach below. According to this approach, the $z$-factor of the gravitational red shift in the case of the Schwarzschild metric is:

$$
z=\frac{f_{E L}}{f_{O}}-1=\frac{f_{\left(r_{0}\right)}^{*}}{f_{\infty}}-1=\frac{f_{\left(r_{0}\right)}^{*}}{f_{\left(r_{0}\right)}}-1=\left(1-\frac{r_{S}}{r_{0}}\right)^{-\frac{1}{2}}-1 ; z \approx \frac{r_{S}}{2 r}=\frac{G M}{c^{2} r_{0}} .
$$

The above exact formula and approximate formula (in the case of a large distance from the center of gravity) derive from the additional assumption that:

$$
f_{\left(r_{0}\right)}^{*}=f_{E L}
$$

which is based on the fact that the energy levels of atoms are not affected by the gravitation (even on the horizon, where the protons and electrons are unmoved). This consideration is also valid only approximately. The application of formula (242) to the Sun's surface $\left\{r_{0}=6.9599 \times 10^{8} \mathrm{~m}, M=1,988,500 \times 10^{24} \mathrm{~kg}[29], G=6.67428(67) \times\right.$ $10^{-11} \mathrm{~m}^{3} \mathrm{~kg}^{-1} \mathrm{~s}^{-2}$, and $c=299,792,458 \mathrm{~ms}^{-1}$ (exact) [28] (pp. 1-1, 1-20, 14-2)\} gives $z_{\text {theoretical }}=2.12244 \times 10^{-6}$. The observation of the 74 strong lines of the iron's spectrum $\mathrm{Fe}(\mathrm{I})$ gives accuracy

$$
R=z_{\text {observed }} / z_{\text {theoretical }}=0.97 \pm 0.16 \quad\left(E_{\mathrm{r}}=-3 \%\right),
$$

while all the 738 (weak, medium, and strong) lines have accuracy [31] (p. 247)

$$
R=z_{\text {observed }} / z_{\text {theoretical }}=0.76 \pm 0.24 \quad\left(E_{\mathrm{r}}=-24 \%\right) .
$$

Note that the above mainstream consideration contains two approximations. So, it is valid only for weak gravitational fields. This could be the cause for the fact that the above observed gravitational red shift is smaller than the corresponding theoretical one. It is 
also reminded that the anomalous gravitational red shift from type Ia distant supernovae depends on the distance of the source from the observer. The corresponding variation is a necessity for Dark Energy [32] (pp. 4-6).

In the case of SR, the gravitational red shift is explained via a different way. For simplicity reasons, we consider the radial motion of the photon. As mentioned above, the electro-magnetic wave has a variable period/frequency, because the photon speed is also variable around the center of gravity $\mathrm{O}$ (see formula (71i)). So, this wave is given by another differential equation/formula than the usual waves with steady speed

$$
\frac{\partial^{2} \Psi}{\partial t^{2}}-c^{2} \frac{\partial^{2} \Psi}{\partial x^{2}}=0 ; \Psi=A \sin \frac{2 \pi}{\lambda_{\infty}}\left(c t \mp x+\phi_{0}\right),
$$

where $A$ is the amplitude of vibration, $\varphi_{0}$ is the initial phase, and the minus/plus sign is referred to the wave moving toward the right/left hand. Additionally, the wavelength is denoted by $\lambda_{\infty}$, because the electro-magnetic wave around the center of gravity $\mathrm{O}$ has the above form at an infinite distance. There exist many formulas for the differential equation of a variable-speed wave [33] (pp. 496-97):

$$
\frac{\partial^{2} \Psi}{\partial t^{2}}-c_{(x)}{ }^{2} \frac{\partial^{2} \Psi}{\partial x^{2}}=0 ; \frac{\partial^{2} \Psi}{\partial t^{2}}-\frac{\partial}{\partial x}\left[c_{(x)}{ }^{2} \frac{\partial \Psi}{\partial x}\right]=0,
$$

The attention here is given on the characteristics of the wave (period/frequency and wavelength), rather than the solution of the differential equation that gives it, which must be covariant under the Lorentz boost and generally under the Lorentz transformation. Considering a ray of light (electro-magnetic wave) emitted from a source at distance $r_{0}$ from the center of gravity $\mathrm{O}$, we have

$$
\mathrm{d} t=\frac{\mathrm{d} r}{c_{(r)}}
$$

whose integral for one period of the vibration $T_{\left(r_{0}\right)}=t_{\left(r_{1}\right)}-t_{\left(r_{0}\right)}$ is

$$
\int_{t_{\left(r_{0}\right)}}^{t_{\left(r_{0}\right)}+T_{\left(r_{0}\right)}} \mathrm{d} t=\int_{r_{0}}^{r_{0} \pm \lambda_{\left(r_{0}\right)}} \frac{\mathrm{d} r}{c_{(r)}}
$$

where $r_{1}-r_{0}= \pm \lambda_{\left(r_{0}\right)}$ is the displacement of the peak within one period of the vibration at $r=r_{0}, \lambda_{\left(r_{0}\right)}$ its wavelength, while the \pm sign signifies whether the photon moves away from $\mathrm{O}$ or approaches it. The above equation in combination with (71i) gives

$$
\int_{t_{\left(r_{0}\right)}}^{t_{\left(r_{0}\right)}+T_{\left(r_{0}\right)}} \mathrm{d} t=\frac{1}{c} \int_{r_{0}}^{r_{0} \pm \lambda_{\left(r_{0}\right)}} \pm \frac{d r}{1-\frac{r_{S}}{r}}= \pm \frac{1}{c}\left[r+r_{S} \ln \left(r-r_{S}\right)\right]_{r_{0}}^{r_{0} \pm \lambda_{\left(r_{0}\right)}},
$$

or equivalently,

$$
T_{\left(r_{0}\right)}= \pm \frac{1}{c}\left[ \pm \lambda_{\left(r_{0}\right)}+r_{S} \ln \frac{r_{0} \pm \lambda_{\left(r_{0}\right)}-r_{S}}{r_{0}-r_{S}}\right]=\frac{\lambda_{\left(r_{0}\right)}}{c}\left[1 \pm \frac{r_{S}}{\lambda_{\left(r_{0}\right)}} \ln \left(1 \pm \frac{\lambda_{\left(r_{0}\right)}}{r_{0}-r_{S}}\right)\right] .
$$

The usual electro-magnetic waves validate the condition

$$
\lambda_{\left(r_{0}\right)} \ll r_{0}-r_{S},
$$


which allows for the following simpler form of (249):

$$
T_{\left(r_{0}\right)} \approx \frac{\lambda_{\left(r_{0}\right)}}{c}\left[1+\frac{r_{S}}{\lambda_{\left(r_{0}\right)}} \frac{\lambda_{\left(r_{0}\right)}}{r_{0}-r_{S}}\right]=\frac{\lambda_{\left(r_{0}\right)}}{c}\left[1+\frac{1}{\frac{r_{0}}{r_{S}}-1}\right],
$$

from where

$$
\lambda_{\left(r_{0}\right)} \approx \frac{c T_{\left(r_{0}\right)}}{1+\frac{1}{\frac{r_{0}}{r_{S}}-1}}=\frac{c}{f_{\left(r_{0}\right)}\left(1+\frac{1}{\frac{r_{0}}{r_{S}}-1}\right)} .
$$

In the case of large distances from the center of gravity $\mathrm{O}$

$$
r_{0} \gg r_{S},
$$

Equation (252) is further simplified to

$$
\lambda_{\left(r_{0}\right)} \approx \frac{c T_{\left(r_{0}\right)}}{1+\frac{r_{S}}{r_{0}}}=\frac{c}{f_{\left(r_{0}\right)}\left(1+\frac{r_{S}}{r_{0}}\right)} .
$$

Of course, when $r_{0} \rightarrow \infty$, (254) is simplified even further and gives the fundamental law of waves with steady speed:

$$
\lambda_{\infty}=c T_{\infty}=\frac{c}{f_{\infty}} .
$$

Moreover, the following formulas can be used for the period/frequency of the variable speed of an electro-magnetic wave:

$$
T_{\left(r_{0}\right)}=\sqrt{1-\frac{r_{S}}{r_{0}}} T_{\infty} ; f_{\left(r_{0}\right)}=\frac{f_{\infty}}{\sqrt{1-\frac{r_{S}}{r_{0}}}} .
$$

Thus, the deriving SR $z$-factor of the gravitational red shift is the same as the above mainstream GR approach (242i):

$$
z=\frac{f_{E L}}{f_{O}}-1=\frac{f_{\left(r_{0}\right)}}{f_{\infty}}-1=\left(1-\frac{r_{S}}{r_{0}}\right)^{-\frac{1}{2}}-1 .
$$

The above formula (in the case of a large distance from the center of gravity $\mathrm{O}$ ), derives from the additional assumption that:

$$
f_{\left(r_{0}\right)}=f_{E L}
$$


which is based on the fact that the energy levels of atoms are not affected by the gravitation (even on the horizon, where the protons and electrons are unmoved). Moreover, the substitution of (256) and (255) to (254) gives

$$
\lambda_{\left(r_{0}\right)} \approx \frac{\sqrt{1-\frac{r_{S}}{r_{0}}} c T_{\infty}}{1+\frac{r_{S}}{r_{0}}}=\frac{\sqrt{1-\frac{r_{S}}{r_{0}}}}{1+\frac{r_{S}}{r_{0}}} \lambda_{\infty},
$$

Furthermore, the corresponding GR total energies of the photon are correlated via the multiplication of (256ii) by h:

$$
h f_{\left(r_{0}\right)}=E_{G R}\left(1-\frac{r_{S}}{r_{0}}\right)^{-\frac{1}{2}}=E_{G R\left(r_{0}\right)}^{*}
$$

where

$$
E_{G R}=E^{*}=h f_{\infty} .
$$

The above formula is in accordance with the maintenance of energy of the system (a spherical body with mass $M$ and a photon with $m=0$ ). Evidently, Equations (57) and (239) are obtained once again. This justifies the use of formula (256).

\section{The Reverse Procedure: GR Metrics from the SR Gravitational Central Scalar Generalized Potential and the Case Study of a Non-Riemannian Metric from the Newtonian Gravitational Potential}

The procedure of correlation of GR metrics with the SR Gravitational Central Scalar Generalized Potential can be reversed. Thus, if we substitute the SR gravitational central scalar generalized potential (160) in formula (158ii), then the Schwarzschild GR time dilation (50) and the GR (Riemannian) Schwarzschild metric (22) are obtained. However, if we start from the Newtonian Gravitational Potential (3) according to SR, then the corresponding metric of GR is non-Riemannian. Indeed, the substitution of (3) in (158ii) implies

$$
\frac{\mathrm{d} t}{\mathrm{~d} \tau_{G R}}=\frac{1}{-\frac{G M}{c^{2} r}+\frac{1}{\gamma_{\left(\beta_{P}\right)}}}=\frac{1}{-\frac{r_{S}}{2 r}+\sqrt{1-\frac{\dot{r}^{2}+r^{2} \dot{\theta}^{2}+r^{2} \sin ^{2} \theta \dot{\phi}^{2}}{c^{2}}}} ;=\frac{\mathrm{d}}{\mathrm{d} t^{\prime}}
$$

or equivalently,

$$
\begin{aligned}
& \left(\frac{\mathrm{d} \tau_{G R}}{\mathrm{~d} t}\right)^{2}=\frac{r_{S}^{2}}{4 r^{2}}+1-\frac{1}{c^{2}}\left[\left(\frac{\mathrm{d} r}{\mathrm{~d} t}\right)^{2}+r^{2}\left(\frac{\mathrm{d} \theta}{\mathrm{d} t}\right)^{2}+r^{2} \sin ^{2} \theta\left(\frac{\mathrm{d} \phi}{\mathrm{d} t}\right)^{2}\right]- \\
& -\frac{r_{S}}{r} \sqrt{1-\frac{1}{c^{2}}\left[\left(\frac{\mathrm{d} r}{\mathrm{~d} t}\right)^{2}+r^{2}\left(\frac{\mathrm{d} \theta}{\mathrm{d} t}\right)^{2}+r^{2} \sin ^{2} \theta\left(\frac{\mathrm{d} \phi}{\mathrm{d} t}\right)^{2}\right]}
\end{aligned}
$$

The above equation gives the non-Riemannian metric of GR:

$$
\begin{aligned}
\mathrm{d} S^{2} & =-c^{2} \mathrm{~d} \tau_{G R}^{2}= \\
& =-\left(1-\frac{r_{S}}{r} \sqrt{1-\frac{1}{c^{2}}\left[\left(\frac{\mathrm{d} r}{\mathrm{~d} t}\right)^{2}+r^{2}\left(\frac{\mathrm{d} \theta}{\mathrm{d} t}\right)^{2}+r^{2} \sin ^{2} \theta\left(\frac{\mathrm{d} \phi}{\mathrm{d} t}\right)^{2}\right]}+\frac{r_{S}^{2}}{4 r^{2}}\right) c^{2} \mathrm{~d} t^{2}+ \\
& +\mathrm{d} r^{2}+r^{2} \mathrm{~d} \theta^{2}+r^{2} \sin ^{2} \theta \mathrm{d} \phi^{2}
\end{aligned}
$$


which produces the 'Finsler' geometry [8] (p. 173) and gives the same results as the Newtonian Gravitational Potential (3) according to SR. Thus, the approach described in this paper can be applied to any gravity theory (Riemannian and/or non-Riemannian) with the use of the corresponding time dilation. So, the Lorentz Gauge Theory [9-14], Teleparallel Gravity [15-21], etc., can be used. In the case of small velocities of the test particle, or equivalently, $r \gg r_{S}$, the above non-Riemannian metric (264) becomes Riemannian:

$$
\mathrm{d} S^{2}=-c^{2} \mathrm{~d} \tau_{G R}{ }^{2}=-\left(1-\frac{r_{S}}{2 r}\right)^{2} c^{2} \mathrm{~d} t^{2}+\mathrm{d} r^{2}+r^{2} \mathrm{~d} \theta^{2}+r^{2} \sin ^{2} \theta \mathrm{d} \phi^{2} ; r \gg r_{S},
$$

but it is not in accordance with Einstein's field equations in a vacuum and the corresponding conditions (17). The above procedure proves that SR can be used for the description of the gravitational interaction combined with the suitable SR Gravitational Scalar Generalized Potential. This potential is completely free of conditions, because formula (159) implies the relativistic force $(\vec{F})$, while the corresponding four-force in the rest frame of the spherical body (RIO O) is given by [2] (pp. 329, 342):

$$
f^{i}=\gamma_{\left(\vec{v}_{P}\right)}\left[\begin{array}{c}
\frac{1}{\vec{F}} \cdot \vec{v}_{P} \\
\vec{F}
\end{array}\right]
$$

The four-force in any other frame (RIO O') is obtained with the use of the Lorentz transformation (SR relativization). So, the SR approach of gravitation can describe the gravitational interaction of GR field equations (original or new) [21], but it is not limited to them.

\section{Discussion}

In the context of SR, the gravity can be treated as a field in spacetime, in contrast to GR where it is a property of the spacetime (curvature and/or torsion) $[9,21]$. This is the only approach in a Minkowski space $\left(\mathrm{M}^{4}\right)$ with constant null curvature $(K=0)$, which is enriched with the Lorentz metric and it is exactly the same as in the case of the corresponding electro-magnetic field. Moreover, according to the bibliography, SR cannot explain all the gravitational phenomena that the Schwarzschild metric can explain, i.e., (i) the precession of Mercury's Perihelion due to the Sun's gravity; (ii) the Gravitational Deflection of Light; (iii) the Shapiro Time Delay; and (iv) the Gravitational Red Shift. This paper proves that this is not true when the suitable gravitational generalized potential (160) is used.

Furthermore, in SR, the gravitational generalized potential can be arbitrary, in contrast to the metrics of GR, which derive from Einstein's field equations and/or the corresponding field equations of new GR [15,21]. The SR Gravitational Scalar Generalized Potential is completely free of conditions, because formula (159) implies the relativistic force and the corresponding four-force in the rest frame of the spherical body (RIO O) is given by (266). The four-force in any other frame (RIO $\mathrm{O}^{\prime}$ ) is obtained with the use of the Lorentz transformation (SR relativization). This is an advantage of SR, as any SR gravitational generalized potential can be used for the explanation of the experimental data in hand, while in GR only the metrics that are in accordance with Einstein's field equations and/or the corresponding field equations of new GR can be used. The dark matter and dark energy hypotheses show that the SR approach of a gravitational field can be useful [32]. Besides, the SR approach to gravity may also be useful for the concept of a unified description of the basic physical interactions, where the biggest barrier is the GR approach to gravity [9].

A number of solutions to gravitational problems have appeared during the first century of GR's existence. The Schwarzschild solution and the corresponding Teleparallel Gravity are used in this paper for the corresponding SR gravitational generalized potential. 
This method may have many more applications. For instance, the potential (160) can be modified as follows:

$$
V_{G S R(r, \dot{r}, \dot{\phi})}=\frac{c^{2}}{\xi_{I}^{2}}\left(\xi_{I}^{2}\left[1-\left(\frac{r_{S}}{r}+\frac{1}{1-\frac{r_{S}}{r}} \frac{\dot{r}^{2}}{c^{2}}+\frac{r^{2} \dot{\phi}^{2}}{c^{2}}\right)\right]^{\frac{1}{2}}-\frac{1}{\gamma_{\left(\xi_{I} \beta_{P}\right)}}\right) ; \cdot=\frac{\mathrm{d}}{\mathrm{d} t}
$$

The above potential cannot be produced by a GR time dilation in accordance with Einstein's field equations (or new GR field equations [21]) and the scalar relativistic potential, but it clarifies the aforementioned gravitational phenomena by using Classical Mechanics $\left(\xi_{I} \rightarrow 0\right)$ or SR $\left(\xi_{I}=1\right)$ [24] (p. 28). This can be regarded as a modification of the classical Newtonian potential with relativistic effects taken into account and adjustment to the Schwarzschild solution known in GR.

In GR, the work is usually done in curved pseudo-Riemannian spaces and especially in those that are reduced locally to Minkowski spacetime [8] (p. 175). Thus, this method can use the time dilation in spaces with the Kerr metric or the Kerr-Newman metric, etc., in order to obtain the corresponding SR gravitational generalized potential.

In addition, the procedure of the correlation of GR metrics with the SR Gravitational Central Scalar Generalized Potential can be reversed. Thus, if the gravitational central scalar generalized potential (160) according to SR is used, then the Schwarzschild metric (22) is obtained (this is a Riemannian metric). However, if we start from the Newtonian Gravitational Potential (3) according to SR, then the corresponding metric of GR (264) is non-Riemannian. Thus, only GR in accordance with 'Finsler' geometry [8] (p. 173) can produce the same results as the Newtonian Gravitational Potential (3) according to SR.

The approach described in this paper can be applied to any theory of gravity (Riemannian and/or non-Riemannian) with the use of the corresponding time dilation. So, the Lorentz Gauge Theory [9-14], Teleparallel Gravity [15-21], etc., can be used. More specifically, Teleparallel Gravity is a gravitational theory formulated on the Weitzenböck spacetime, characterized by the vanishing curvature tensor (absolute parallelism) and by the torsion tensor formed of four parallel vector fields. This theory is called new general relativity because Albert Einstein in 1928 first gave its original form [15,21] (p. 3524). The corresponding isotropic metric by a static, spherical body (assuming that the spin of constituent particles of a body can be completely neglected) is given by (210), which contains constant parameters $p=p(\varepsilon) ; q=q(\varepsilon)$, where $\varepsilon$ is an arbitrary parameter with an experimental low value (220). When $\varepsilon \rightarrow 0$, then $p=q=2$ and metric (210) becomes the (Riemannian) isotropic form of the Schwarzschild metric (27). This happens because the field equations in Weitzenbock spacetime are reduced to Einstein's field equations under two conditions: (i) the axial-vector part of the torsion tensor vanishes identically; and (ii) the effects due to the intrinsic spin $-1 / 2$ fundamental particles can be neglected [21] (p. 3539). When $\varepsilon \rightarrow 0(p=q=2)$, then the corresponding SR gravitational generalized potential is the SR Schwarzschild Generalized Potential given by formula (160). In any other case $\varepsilon \neq 0(p \neq 2, q \neq 2)$, we calculate the metric by a static, spherical body (assuming that the spin of constituent particles of a body can be completely neglected) (232) and the corresponding time dilation (234) in Weitzenböck spacetime. The substitution of the time dilation (234) to formula (158) gives the corresponding SR Teleparallel Gravity Generalized Potential (235). We observe that the GR Schwarzschild time dilation (50) and SR Schwarzschild Generalized Potential (160) can be regarded as the limit of the corresponding quantities of Teleparallel Gravity (234) and (235) for $\varepsilon \rightarrow 0(p=q \rightarrow 2)$ (assuming that the spin of constituent particles of a static spherical body is completely neglected).

Finally, it is noted that a weak field approximation is used in the standard GR in order to obtain the gravitational redshift due to a spherical mass. This approach is not mentioned in the bibliography, which considers that the standard Schwarzschild gravitational redshift is valid in strong gravitational fields as well. This fact could be the cause for the smaller 
observed gravitational red shift in the solar spectrum than the corresponding theoretical one $\left(E_{\mathrm{r}}=-3 \%\right.$ for the strong lines of the iron's spectrum Fe(I)) [31].

\section{Conclusions}

The gravitational field can be described equally well via either variable metrics of spacetime according to General Relativity (GR), or Gravitational Generalized Potential according to Special Relativity (SR). In this paper, the GR gravitational results are obtained by using $S R$ via the SR Lagrangian, which contains the corresponding GR time dilation. On the scale of a black hole, a planetary system, or a star system, the Precession of Mercury's perihelion, the Gravitational Deflection of Light, the Shapiro time delay, and the Gravitational Red Shift can be explained equally well with either the Schwarzschild metric according to GR, or the SR Schwarzschild Generalized Potential (160), according to SR. The aforementioned can also be explained by using the suitable Gravitational Generalized Potential (267) for $\xi_{I} \rightarrow 0$ according to Newtonian Physics.

The above procedure can be reversed. Thus, if the gravitational central scalar generalized potential (160) according to SR is used, then the Schwarzschild metric is obtained (this is a Riemannian metric). In contrast, the Newtonian Gravitational Potential (3) according to SR leads to the corresponding non-Riemannian metric (264) of GR. So, the approach described in this paper can be applied to any gravity theory (Riemannian and/or non-Riemannian), such as Lorentz Gauge Theory [9-14], Teleparallel Gravity [15-21], etc., with the use of the corresponding time dilation.

In this way, the work with the gravitational fields can be done in the same manner as in any other type of field (such as the electro-magnetic) in Minkowski spacetime with the Lorentz Metric, avoiding thus dealing with the motions of particles in the curved spacetime of GR. This may also be useful for the concept of a unified description of the basic physical interactions, where the biggest barrier is the gravity according to GR [9]. Finally, it is shown that the mainstream consideration of the Gravitational Red Shift contains two approximations that are valid in weak gravitational fields only.

Author Contributions: The research paper (original theory, mathematical calculations, data analysis, etc.) was equally developed by S.V., E.V. and C.G.M. S.V. and E.V. wrote the draft, which C.G.M. reviewed and corrected. All authors have read and agreed to the published version of the manuscript.

Funding: This research received no external funding. The APC was funded by the MDPI journal Particles.

Institutional Review Board Statement: Not applicable.

Informed Consent Statement: Not applicable.

Data Availability Statement: Not applicable.

Conflicts of Interest: The authors declare no conflict of interest.

\section{Abbreviations}

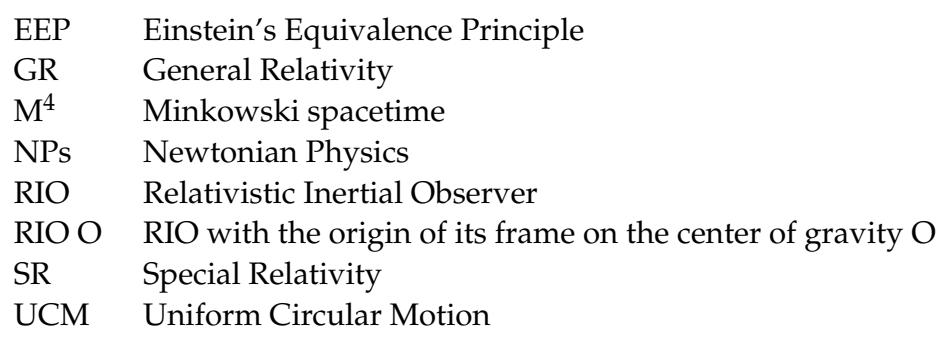

\section{References}

1. Phipps, T.E. Mercury's precession according to special relativity. Am. J. Phys. 1986, 54, 245-247. [CrossRef]

2. Tsamparlis, M. Special Relativity: An Introduction with 200 Problems and Solutions, 1st ed.; Springer: Berlin/Heidelberg, Germany, 2010; ISBN 978-3-642-03836-5. 
3. Goldstein, H. Classical Mechanics, 2nd ed.; Addison-Wesley: London, UK, 1980; ISBN 0-201-02969-3.

4. Park, R.S.; Folkner, W.M.; Konopliv, A.S.; Williams, J.G.; Smith, D.E.; Zuber, M.T. Precession of Mercury's Perihelion from Ranging to the MESSENGER Spacecraft. Astron. J. 2017, 153, 121. [CrossRef]

5. Einstein, A. Relativity: The Special and General Theory; Robert, W.L., Translator; Henry Holt: New York, NY, USA, 1920.

6. von Soldner, J.G. Ueber die Ablenkung eines Lichtstrals von seiner geradlinigen Bewegung, durch die Attraktion eines Weltkörpers, an welchem er nahe vorbei geht. Berl. Astron. Jahrb. 1804, 1804, 161-172.

7. Einstein, A. On the Influence of Gravitation on the Propagation of Light. Ann. Phys. 1911, 35, 898-908. doi: 10.1002/andp.19113401005 [CrossRef]

8. Rindler, W. Relativity: Special, General and Cosmological, 2nd ed.; Oxford University Press: New York, NY, USA, 2006; ISBN 978-0-19-856732-5.

9. Blagojevic, M. Gravitation and Gauge Symmetries; IOP Publishing: Bristol, UK, 2002; ISBN 0750307676.

10. Utiyama, R. Invariant Theoretical Interpretation of Interaction. Phys. Rev. 1956, 101, $1597-1607$. doi: 10.1103/PhysRev.101.1597 [CrossRef]

11. Kibble, T.W.B. Lorentz Invariance and the Gravitational Field. J. Math. Phys. 1961, 2, 212-221. [CrossRef]

12. Lecian, O.M.; Mercuri, S.; Montani, G. Is Torsion a Fundamental Physical Field? In Proceedings of the 11th Marcel Grossmann Meeting on General Relativity, Berlin, Germany, 23-29 July 2006; World Scientific: Singapore, 2008; pp. 2668-2670. [CrossRef]

13. Lecian, O.M.; Montani, G.; Carlevaro, N. Novel analysis of spinor interactions and non-Riemannian geometry. Eur. Phys. J. Plus 2013, 128, 19. [CrossRef]

14. Hehl, F.W.; Von Der Heyde, P.; Kerlick, G.D.; Nester, J.M. General relativity with spin and torsion: Foundations and prospects. Rev. Mod. Phys. 1976, 48, 393-416. [CrossRef]

15. Einstein, A. Riemann-Geometrie mit Aufrechterhaltung des Begriffes des Fernparallelismus; Sitzungsberichte, K., Ed.; Preussische Akademie der Wissenschaften, Phys-math: Berlin, Germany, 1928; pp. 217-221.

16. Møller, C. Conservation laws and absolute parallelism in general relativity. Mat. Fys. Dan. Vid. Selsk. 1961, 1, 50.

17. Pellegrini, C.; Plebanski, J. Tetrad fields and gravitational fields. Mat. Fys. SKR. Dan. Vid. Selsk. 1963, 2, 1-39.

18. Maluf, J.W. The Teleparallel Equivalent of General Relativity and the Gravitational Centre of Mass. Universe 2016, 2, 19. [CrossRef]

19. Perlick, V. Characterization of standard clocks by means of light rays and freely falling particles. Gen. Relativ. Gravit. 1987, 19, 1059-1073. [CrossRef]

20. Delhom, A.; Lobo, I.P.; Olmo, G.J.; Romero, C. Conformally invariant proper time with general non-metricity. Eur. Phys. J. C 2020, 80, 415. [CrossRef]

21. Hayashi, K.; Shirafuji, T. New General Relativity. Phys. Rev. D 1979, 19, 12, 3524-3553. [CrossRef]

22. Golovko, V. New metrics for the gravitational field of a point mass. Results Phys. 2019, 13, 102288. [CrossRef]

23. Schwarzschild, K. Über das Gravitationsfeld eines Massenpunktes nach der Einsteinschen Theorie. Sitz. Königlich Preuss. Akad. Wiss. 1916, 7, 189-196.

24. Vossos, S.; Vossos, E.; Massouros, C.G. Explanation of Light Deflection, Precession of Mercury's Perihelion, Gravitational Red Shift and Rotation Curves in Galaxies, by using General Relativity or equivalent Generalized Scalar Gravitational Potential, according to Special Relativity and Newtonian Physics. J. Phys. Conf. Ser. 2021, 1730, 012080. [CrossRef]

25. Reasenberg, R.D.; Shapiro, I.I.; MacNeil, P.E.; Goldstein, R.B.; Breidenthal, J.C.; Brenkle, J.P.; Cain, D.L.; Kaufman, T.M.; Komarek, T.A.; Zygielbaum, A.I. Viking relativity experiment-Verification of signal retardation by solar gravity. Astrophys. J. 1979, 234, L219-L221. [CrossRef]

26. Vossos, S.; Vossos, E.; Massouros, C.G. New Central Scalar Gravitational Potential according to Special Relativity and Newtonian Physics, explains the Precession of Mercury's Perihelion, the Gravitational Red Shift and the Rotation Curves in Galaxies, eliminating Dark Matter. J. Phys. Conf. Ser. 2019, 1391, 012095. [CrossRef]

27. Williams, D.R. Mercury Fact Sheet. Available online: https://nssdc.gsfc.nasa.gov/planetary/factsheet/mercuryfact.html (accessed on 17 June 2020).

28. Lide, D.R. (Ed.) CRC Handbook of Chemistry and Physics, 89th ed.; Internet Version; CRC Press/Taylor and Francis: Boca Raton, FL, USA, 2009.

29. Williams, D.R. Sun Fact Sheet. Available online: https://nssdc.gsfc.nasa.gov/planetary/factsheet/sunfact.html (accessed on 17 June 2020).

30. Williams, D.R. Earth Fact Sheet. Available online: https://nssdc.gsfc.nasa.gov/planetary/factsheet/earthfact.html (accessed on 17 June 2020).

31. Lopresto, J.C.; Chapman, R.D.; Sturgis, E.A. Solar gravitational redshift. Sol. Phys. 1980, 66, 245-249. [CrossRef]

32. Heymans, C. The Dark Universe; IOP Publishing: Bristol, UK, 2017; ISBN 978-0-7503-1373-5. [CrossRef]

33. Grimshaw, R.; Pelinovsky, D.; Pelinovsky, E. Homogenization of the variable-speed wave equation. Wave Motion 2010, 47, 496-507. [CrossRef] 